\title{
THE STRUCTURE OF BALANCED MULTIVARIATE BIORTHOGONAL MULTIWAVELETS AND DUAL MULTIFRAMELETS
}

\author{
BIN HAN
}

\begin{abstract}
Multiwavelets and multiframelets are of interest in several applications such as numerical algorithms and signal processing, due to their desirable properties such as high smoothness and vanishing moments with relatively small supports of their generating functions and masks. In order to process and represent vector-valued discrete data efficiently and sparsely by a multiwavelet transform, a multiwavelet has to be prefiltered or balanced. Balanced orthonormal univariate multiwavelets and multivariate biorthogonal multiwavelets have been studied and constructed in the literature. Dual multiframelets include (bi)orthogonal multiwavelets as special cases, but their fundamental prefiltering and balancing property has not yet been investigated in the literature. In this paper we shall study the balancing property of multivariate multiframelets from the point of view of the discrete multiframelet transform. This approach, to our best knowledge, has not been considered so far in the literature even for multiwavelets, but it reveals the essential structure of prefiltering and the balancing property of multiwavelets and multiframelets. We prove that every biorthogonal multiwavelet can be prefiltered with the balancing order matching the order of its vanishing moments; that is, from every given compactly supported multivariate biorthogonal multiwavelet, one can always build another (essentially equivalent) compactly supported biorthogonal multiwavelets with its balancing order matching the order of the vanishing moments of the original one. More generally, we show that if a dual multiframelet can be prefiltered, then it can be equivalently transformed into a balanced dual multiframelet with the same balancing order. However, we notice that most available dual multiframelets in the literature cannot be simply prefiltered with its balancing order matching its order of vanishing moments and they must be designed to possess high balancing orders. The key ingredient of our approach is based on investigating some properties of the subdivision and transition operators acting on discrete vector polynomial sequences, which play a central role in a discrete multiframelet transform and are of interest in their own right. We also establish a new canonical form of a matrix mask, which greatly facilitates the investigation and construction of multiwavelets and multiframelets. In this paper, we obtain a complete criterion and the essential structure for balanced or prefiltered dual multiframelets in the most general setting. Our investigation of the balancing property of a multiframelet deepens our understanding of the multiframelet transform in signal processing and scientific computation.
\end{abstract}

Received by the editor July 21, 2008 and, in revised form, April 22, 2009 2010 Mathematics Subject Classification. Primary 42C40, 65T60, 94A08.

Key words and phrases. A discrete multiframelet transform, balancing property, prefiltering, dual multiframelets, biorthogonal multiwavelets, sum rules, vanishing moments.

This research was supported in part by NSERC Canada under Grant RGP 228051. 


\section{INTRODUCTION AND MOTIVATION}

Multiwavelets and multiframelets are derived from refinable function vectors via a multiresolution analysis and are of interest in several applications such as signal processing, numerical algorithms, and computer graphics. In order to process and represent vector-valued discrete data efficiently and sparsely by a discrete multiwavelet transform, a multiwavelet must be either prefiltered or balanced in advance, due to the discrepancy of its approximation properties between the function setting and the discrete vector data setting. Balanced or prefiltered orthonormal univariate multiwavelets have been studied and constructed in the literature. The reader is referred to [1, 3, 4, 14, 15, 18, 19, 20] and the references therein for a comprehensive discussion on the background, motivation, and literature on balanced multiwavelets. Later in this section, we shall give a detailed explanation of the importance of the balancing property of multiwavelets and multiframelets from the point of view of a discrete multiframelet transform instead of the traditional function setting.

Recently there has been a growing interest in the study and construction of framelets and multiframelets; see [2, 6, 7, 10, 11, 12, 13, 16, 17, and the many references therein. For example, tight and dual multiframelets have been characterized in [7, 16, 17] and a unitary extension principle has been given in [16, 17] for constructing dual framelets from scalar refinable functions. More recently, an oblique extension principle has been proposed in [6] (also cf. [2] and [5, 11, 13]) for constructing dual multiframelets with high vanishing moments from refinable function vectors. A multiframelet includes a multiwavelet as a special case by allowing redundancy into a wavelet system. As demonstrated in [2, 6, 11, 13, multiframelets have more freedom in their design and the redundancy in a multiframelet is a desirable feature in several applications such as signal denoising and numerical algorithms. In order to process and represent vector-valued discrete data efficiently and sparsely by a discrete multiframelet transform, the multiframelet must be similarly either prefiltered or balanced in advance. Except for some introductory discussions in [11] on univariate dual multiframelets, this fundamental issue on the balancing and prefiltering property of a multivariate multiframelet has not been addressed so far in the literature. Moreover, to our best knowledge, all approaches in the literature to studying the balancing property of a multiwavelet are from the point of view of the function setting. In this paper, instead we shall study the balancing and approximation properties of multivariate multiframelets from the point of view of a discrete multiframelet transform. We shall see in this paper that it is more natural to study and understand the balancing property of multiframelets from the point of view of a discrete data setting than the function setting. Our results not only generalize the results in the literature (e.g., [1, 3, 4, 11, 14, 15, 18, 19, 20]) on balanced biorthogonal multiwavelets to multivariate dual multiframelets, but also shed a new light on understanding the balancing property of (bi)orthogonal multiwavelets.

Before proceeding further, we first recall some notation and definitions. We say that a $d \times d$ integer matrix $M$ is a dilation matrix if $\lim _{n \rightarrow \infty} M^{-n}=0$; that is, all the eigenvalues of $M$ are greater than 1 in modulus. An $M$-refinable function (or distribution) vector $\phi=\left(\phi_{1}, \ldots, \phi_{r}\right)^{T}$ satisfies the vector refinement equation

$$
\phi=|\operatorname{det} M| \sum_{k \in \mathbb{Z}^{d}} a_{k} \phi(M \cdot-k)
$$


where $a=\left\{a_{k}\right\}_{k \in \mathbb{Z}^{d}}: \mathbb{Z}^{d} \rightarrow \mathbb{C}^{r \times r}$ is called a (matrix) mask with multiplicity $r$ for $\phi$. For a sequence $u=\left\{u_{k}\right\}_{k \in \mathbb{Z}^{d}}: \mathbb{Z}^{d} \rightarrow \mathbb{C}^{n \times r}$, its (formal) Fourier series $\hat{u}$ is defined to be

$$
\hat{u}(\xi):=\sum_{k \in \mathbb{Z}^{d}} u_{k} e^{-i k \cdot \xi}, \quad \xi \in \mathbb{R}^{d},
$$

where $k \cdot \xi$ denotes the inner product of the vectors $k$ and $\xi$ in $\mathbb{R}^{d}$. If $u$ is finitely supported, then $\hat{u}$ is a matrix of $2 \pi$-periodic trigonometric polynomials in $d$-variables. Now the refinement equation in (1.1) can be rewritten in the frequency domain as

$$
\hat{\phi}\left(M^{T} \xi\right)=\hat{a}(\xi) \hat{\phi}(\xi), \quad \xi \in \mathbb{R}^{d},
$$

where $M^{T}$ denotes the transpose of the matrix $M$. For $f \in L_{1}\left(\mathbb{R}^{d}\right)$, its Fourier transform $\hat{f}$ is defined to be $\hat{f}(\xi):=\int_{\mathbb{R}^{d}} f(x) e^{-i x \cdot \xi} d x, \xi \in \mathbb{R}^{d}$, which can be naturally extended to tempered distributions. A wavelet function vector $\psi=$ $\left(\psi_{1}, \ldots, \psi_{r}\right)^{T}$ is generally derived from a refinable function vector $\phi$ via $\hat{\psi}\left(M^{T} \xi\right):=$ $\hat{b}(\xi) \hat{\phi}(\xi)$ for some $r \times r$ matrix $\hat{b}$ of $2 \pi$-periodic trigonometric polynomials in $d$ variables with some desirable properties.

In this paper we are interested in investigating the balancing property of MRA dual multiframelets. Let $\left\{\psi^{1}, \ldots, \psi^{L}\right\}$ be a finite set of $r \times 1$ function vectors in $L_{2}\left(\mathbb{R}^{d}\right)$. We say that $\left\{\psi^{1}, \ldots, \psi^{L}\right\}$ generates an $M$-multiframelet in $L_{2}\left(\mathbb{R}^{d}\right)$ if

$$
\left\{\psi_{j, k}^{\ell}:=|\operatorname{det} M|^{j / 2} \psi^{\ell}\left(M^{j} \cdot-k\right): j \in \mathbb{Z}, k \in \mathbb{Z}^{d}, \ell=1, \ldots, L\right\}
$$

is a frame in $L_{2}\left(\mathbb{R}^{d}\right)$, that is, if there exist two positive constants $C_{1}$ and $C_{2}$ such that

$$
C_{1}\|f\|_{L_{2}\left(\mathbb{R}^{d}\right)}^{2} \leqslant \sum_{\ell=1}^{L} \sum_{j \in \mathbb{Z}} \sum_{k \in \mathbb{Z}^{d}}\left|\left\langle f, \psi_{j, k}^{\ell}\right\rangle\right|^{2} \leqslant C_{2}\|f\|_{L_{2}\left(\mathbb{R}^{d}\right)}^{2} \quad \forall f \in L_{2}\left(\mathbb{R}^{d}\right),
$$

where $\left|\left\langle f, \psi_{j, k}^{\ell}\right\rangle\right|^{2}:=\left\langle f, \psi_{j, k}^{\ell}\right\rangle\left\langle\psi_{j, k}^{\ell}, f\right\rangle$ is the square of the $\ell_{2}$ Euclidean norm of the row vector $\left\langle f, \psi_{j, k}^{\ell}\right\rangle$ in $\mathbb{R}^{1 \times r}$. For an $n \times r$ matrix $g$ and an $n^{\prime} \times r$ matrix $h$ of functions in $L_{2}\left(\mathbb{R}^{d}\right)$, the inner product $\langle g, h\rangle$ is an $n \times n^{\prime}$ matrix defined by

$$
\langle g, h\rangle:=\int_{\mathbb{R}^{d}} g(x) \overline{h(x)}^{T} d x, \quad g \in\left(L_{2}\left(\mathbb{R}^{d}\right)\right)^{n \times r}, h \in\left(L_{2}\left(\mathbb{R}^{d}\right)\right)^{n^{\prime} \times r} .
$$

If both $\left\{\psi^{1}, \ldots, \psi^{L}\right\}$ and $\left\{\tilde{\psi}^{1}, \ldots, \tilde{\psi}^{L}\right\}$ generate $M$-multiframelets in $L_{2}\left(\mathbb{R}^{d}\right)$ and satisfy

$$
\langle f, g\rangle=\sum_{\ell=1}^{L} \sum_{j \in \mathbb{Z}} \sum_{k \in \mathbb{Z}^{d}}\left\langle f, \tilde{\psi}_{j, k}^{\ell}\right\rangle\left\langle\psi_{j, k}^{\ell}, g\right\rangle \quad \forall f, g \in L_{2}\left(\mathbb{R}^{d}\right)
$$

where

and

$$
\psi_{j, k}^{\ell}:=|\operatorname{det} M|^{j / 2} \psi^{\ell}\left(M^{j} \cdot-k\right)
$$

$$
\tilde{\psi}_{j, k}^{\ell}:=|\operatorname{det} M|^{j / 2} \tilde{\psi}^{\ell}\left(M^{j} \cdot-k\right),
$$

then we say that $\left(\left\{\psi^{1}, \ldots, \psi^{L}\right\},\left\{\tilde{\psi}^{1}, \ldots, \tilde{\psi}^{L}\right\}\right)$ generates a pair of dual M-multiframelets in $L_{2}\left(\mathbb{R}^{d}\right)$. By (1.6), every function $f \in L_{2}\left(\mathbb{R}^{d}\right)$ has the multiframelet representation:

$$
f=\sum_{\ell=1}^{L} \sum_{j \in \mathbb{Z}} \sum_{k \in \mathbb{Z}^{d}}\left\langle f, \tilde{\psi}_{j, k}^{\ell}\right\rangle \psi_{j, k}^{\ell}
$$


with the series converging absolutely in the $L_{2}$ norm.

Biorthogonal multiwavelets consist of a particular family of dual multiframelets. We say that $\left(\left\{\psi^{1}, \ldots, \psi^{L}\right\},\left\{\tilde{\psi}^{1}, \ldots, \tilde{\psi}^{L}\right\}\right)$ generates a pair of biorthogonal $M$-multiwavelets in $L_{2}\left(\mathbb{R}^{d}\right)$ if it generates a pair of dual $M$-multiframelets in $L_{2}\left(\mathbb{R}^{d}\right)$ and satisfies the biorthogonality condition:

$$
\left\langle\psi_{j, k}^{\ell}, \tilde{\psi}_{j^{\prime}, k^{\prime}}^{\ell^{\prime}}\right\rangle=\delta_{\ell-\ell^{\prime}} \delta_{j-j^{\prime}} \delta_{k-k^{\prime}} I_{r}, \quad j, j^{\prime} \in \mathbb{Z}, k, k^{\prime} \in \mathbb{Z}^{d}, \ell, \ell^{\prime}=1, \ldots, L,
$$

where $I_{r}$ denotes the $r \times r$ identity matrix and $\delta$ denotes the Dirac sequence such that $\delta_{0}=1$ and $\delta_{k}=0$ for all $k \neq 0$.

Let $\hat{a}, \widehat{b^{1}}, \ldots, \widehat{b^{L}}$ and $\hat{\tilde{a}}, \widehat{\tilde{b}^{1}}, \ldots, \widehat{\tilde{b}^{L}}$ be $r \times r$ matrices of $2 \pi$-periodic trigonometric polynomials in $d$ variables such that they satisfy

$$
{\overline{P_{\left[\hat{a}, \widehat{\tilde{b}^{1}}, \ldots, \widehat{\left.\tilde{b}^{L}\right]}\right.}(\xi)}}^{T} P_{\left[\hat{a}, \widehat{b^{1}}, \ldots, \widehat{b}^{L}\right]}(\xi)=I_{m r},
$$

where $m:=|\operatorname{det} M|$ and

$$
P_{\left[\hat{a}, \widehat{b^{1}}, \ldots, \widehat{b^{L}}\right]}(\xi):=\left[\begin{array}{cccc}
\hat{a}\left(\xi+2 \pi \gamma_{0}\right) & \hat{a}\left(\xi+2 \pi \gamma_{1}\right) & \cdots & \hat{a}\left(\xi+2 \pi \gamma_{m-1}\right) \\
\widehat{b^{1}}\left(\xi+2 \pi \gamma_{0}\right) & \widehat{b}^{1}\left(\xi+2 \pi \gamma_{1}\right) & \cdots & \widehat{b}^{1}\left(\xi+2 \pi \gamma_{m-1}\right) \\
\vdots & \vdots & \ddots & \vdots \\
\widehat{b^{L}}\left(\xi+2 \pi \gamma_{0}\right) & \widehat{b^{L}}\left(\xi+2 \pi \gamma_{1}\right) & \cdots & \widehat{b^{L}}\left(\xi+2 \pi \gamma_{m-1}\right)
\end{array}\right]
$$

with $\left\{\gamma_{0}, \ldots, \gamma_{m-1}\right\}=\Gamma_{M^{T}}$ and $\gamma_{0}=0$, where $\Gamma_{M^{T}}$ denotes a complete set of representatives of the distinct cosets of the quotient group $\left[\left(M^{T}\right)^{-1} \mathbb{Z}^{d}\right] / \mathbb{Z}^{d}$ with $0 \in \Gamma_{M^{T}}$.

A pair of dual $M$-multiframelets is generally obtained from a pair of refinable function vectors in $L_{2}\left(\mathbb{R}^{d}\right)$. Suppose that $\phi$ and $\tilde{\phi}$ are two compactly supported $r \times 1 M$-refinable function vectors in $L_{2}\left(\mathbb{R}^{d}\right)$ such that

$$
\hat{\phi}\left(M^{T} \xi\right)=\hat{a}(\xi) \hat{\phi}(\xi), \quad \hat{\tilde{\phi}}\left(M^{T} \xi\right)=\hat{\tilde{a}}(\xi) \hat{\tilde{\phi}}(\xi) \quad \text { with } \quad \overline{\hat{\phi}}(0)^{T} \hat{\tilde{\phi}}(0)=1 .
$$

Define wavelet function vectors $\psi^{1}, \ldots, \psi^{L}$ and dual wavelet function vectors $\tilde{\psi}^{1}$, $\ldots, \tilde{\psi}^{L}$ by

$$
\begin{aligned}
& \widehat{\psi^{\ell}}\left(M^{T} \xi\right):=\widehat{b^{\ell}}(\xi) \hat{\phi}(\xi) \quad \text { and } \\
& \widehat{\tilde{\psi}^{\ell}}\left(M^{T} \xi\right):=\widehat{\tilde{b}^{\ell}}(\xi) \hat{\tilde{\phi}}(\xi), \quad \xi \in \mathbb{R}^{d}, \ell=1, \ldots, L .
\end{aligned}
$$

Now one can directly verify that (1.9) implies that

$$
\begin{aligned}
& \sum_{k \in \mathbb{Z}^{d}}\left\langle f, \tilde{\phi}_{j+1, k}\right\rangle\left\langle\phi_{j+1, k}, g\right\rangle \\
& =\sum_{k \in \mathbb{Z}^{d}}\left\langle f, \tilde{\phi}_{j, k}\right\rangle\left\langle\phi_{j, k}, g\right\rangle+\sum_{\ell=1}^{L} \sum_{k \in \mathbb{Z}^{d}}\left\langle f, \tilde{\psi}_{j, k}^{\ell}\right\rangle\left\langle\psi_{j, k}^{\ell}, g\right\rangle \quad \forall j \in \mathbb{Z}, f, g \in L_{2}\left(\mathbb{R}^{d}\right) .
\end{aligned}
$$

Note that all $\phi, \psi^{1}, \ldots, \psi^{L}, \tilde{\phi}, \tilde{\psi}^{1}, \ldots, \tilde{\psi}^{L}$ are compactly supported function vectors in $L_{2}\left(\mathbb{R}^{d}\right)$. If all $\psi^{1}, \ldots, \psi^{L}$ and $\tilde{\psi}^{1}, \ldots, \tilde{\psi}^{L}$ have at least one vanishing moment; that is,

$$
\widehat{b^{1}}(0) \hat{\phi}(0)=\cdots=\widehat{b^{L}}(0) \hat{\phi}(0)=\widehat{\tilde{b}^{1}} \hat{\tilde{\phi}}(0)=\cdots=\widehat{\tilde{b}^{L}}(0) \hat{\tilde{\phi}}(0)=0,
$$

by [10, Theorem 2.3], the inequality on the right side of (1.4) holds. Similarly, the inequality on the right side of (1.4) also holds when $\psi^{1}, \ldots, \psi^{L}$ are replaced by 
$\tilde{\psi}^{1}, \ldots, \tilde{\psi}^{L}$, respectively. Now it is not difficult to deduce that if (1.9) and (1.14) hold, then $\left(\left\{\psi^{1}, \ldots, \psi^{L}\right\},\left\{\tilde{\psi}^{1}, \ldots, \tilde{\psi}^{L}\right\}\right)$ generates a pair of dual $M$-multiframelets in $L_{2}\left(\mathbb{R}^{d}\right)$. For more details, see [11, Theorem 3.4] and [2, 5, 6, 7, 10, 13, 16, 17.

If in addition $L=|\operatorname{det} M|-1$ and $\phi, \tilde{\phi}$ satisfy the biorthogonality relation

$$
\langle\phi, \tilde{\phi}(\cdot-k)\rangle=\delta_{k} I_{r}, \quad k \in \mathbb{Z}^{d},
$$

then $\left(\left\{\psi^{1}, \ldots, \psi^{L}\right\},\left\{\tilde{\psi}^{1}, \ldots, \tilde{\psi}^{L}\right\}\right)$ generates a pair of biorthogonal $M$-multiwavelets in $L_{2}\left(\mathbb{R}^{d}\right)$. In this case, since $L=|\operatorname{det} M|-1$, the matrix $P_{\left[\hat{a}, \widehat{b^{1}}, \ldots, \widehat{b^{L}}\right]}$ in (1.10) becomes a square matrix of $2 \pi$-periodic trigonometric polynomials in $d$ variables.

For a positive integer $\kappa$, throughout this paper $\Pi_{\kappa}$ denotes the set of all polynomials in $d$ variables of total degree no more than $\kappa$. To have a sparse multiframelet representation in (1.7), an important property of a multiframelet is its order of vanishing moments. We say that $\left\{\tilde{\psi}^{1}, \ldots, \tilde{\psi}^{L}\right\}$ has $\kappa+1$ vanishing moments if

$$
\int_{\mathbb{R}^{d}} h(x) \tilde{\psi}^{\ell}(x) d x=0 \quad \forall \ell=1, \ldots, L \quad \text { and } \quad h \in \Pi_{\kappa} .
$$

When $f$ agrees with a polynomial of degree no more than $\kappa$ inside the support of the function vector $\tilde{\psi}_{j, k}^{\ell}$, by (1.16), we see that the wavelet coefficient $\left\langle f, \tilde{\psi}_{j, k}^{\ell}\right\rangle=0$. In other words, if $f$ can be well approximated by some polynomial of degree no more than $\kappa$ inside the support of $\tilde{\psi}_{j, k}^{\ell}$, then the notion of $\kappa+1$ vanishing moments of $\tilde{\psi}^{1}, \ldots, \tilde{\psi}^{L}$ guarantees that the (high-pass) wavelet coefficients $\left\langle f, \tilde{\psi}_{j, k}^{\ell}\right\rangle$ will be negligible. So, for a smooth function $f$, the multiframelet representation in (1.7) is sparse, which is one of the most desirable features of wavelets and framelets. For the scalar wavelet case (that is, the multiplicity of all wavelet function vectors is $r=1$ ), this desirable property of vanishing moments guarantees that a similar desirable property holds for the discrete wavelet transform in the discrete data setting. In other words, for a discrete datum $u$ which takes sampled values on the integer lattice of a polynomial with degree no more than $\kappa$, the output high-pass wavelet coefficients of $u$, after applying a discrete wavelet transform, are identically zero. However, as we shall see later, for multiplicity $r>1$, this desirable property of wavelets for the function setting is not automatically carried over to the discrete multiframelet transform for discrete vector data. In order to overcome such a difficulty, either a prefilter is required for a given multiframelet or a multiframelet has to be designed in advance to possess some extra balancing property (see [1, 3, 4, 11, 14, 15, 18, 19, 20,).

It is the purpose of this paper to investigate the balancing property of a multivariate multiframelet from the point of view of a discrete algorithm using only the condition in (1.9). This point of view will enable us to understand the essence of the above-mentioned difficulty facing most multiwavelets and multiframelets. This in turn will give us an overall better picture about both prefiltering of multiwavelets and balanced multiframelets.

In order to present the discrete multiframelet transform, we first recall two linear operators acting on discrete vector data - the subdivision and transition operators, whose properties will play a central role in our study of the balancing property of a discrete multiframelet transform.

By $\left(\ell\left(\mathbb{Z}^{d}\right)\right)^{n \times r}$ we denote the linear space of all sequences $u: \mathbb{Z}^{d} \mapsto \mathbb{C}^{n \times r}$, that is, $u=\left\{u_{k}\right\}_{k \in \mathbb{Z}^{d}}$ with each $u_{k}$ being an $n \times r$ matrix of complex numbers. In many applications, instead of a function $f$, information is often recorded in the discrete 
form, that is, a sequence $u \in\left(\ell\left(\mathbb{Z}^{d}\right)\right)^{n \times r}$ which could be obtained by sampling an underlying continuous function $f$. In order to have a similar multiframelet representation in (1.7) for a discrete data, a fast multiframelet transform is used in the literature, which we shall discuss in detail as follows. For a dilation matrix $M$ and a finitely supported sequence $u: \mathbb{Z}^{d} \mapsto \mathbb{C}^{r \times r}$, the subdivision operator $S_{u, M}:\left(\ell\left(\mathbb{Z}^{d}\right)\right)^{n \times r} \mapsto\left(\ell\left(\mathbb{Z}^{d}\right)\right)^{n \times r}$ and the transition operator $T_{u, M}:\left(\ell\left(\mathbb{Z}^{d}\right)\right)^{n \times r} \mapsto$ $\left(\ell\left(\mathbb{Z}^{d}\right)\right)^{n \times r}$ are defined to be

$$
\begin{aligned}
& {\left[S_{u, M} v\right]_{j}:=|\operatorname{det} M| \sum_{k \in \mathbb{Z}^{d}} v_{k} u_{j-M k},} \\
& {\left[T_{u, M} v\right]_{j}:=\sum_{k \in \mathbb{Z}^{d}} v_{k}{\overline{u_{k-M j}}}^{T},} \\
& j \in \mathbb{Z}^{d}, v \in\left(\ell\left(\mathbb{Z}^{d}\right)\right)^{n \times r} .
\end{aligned}
$$

In the frequency domain, one can easily verify that (1.17) is equivalent to

$$
\begin{aligned}
& \widehat{S_{u, M} v}(\xi)=|\operatorname{det} M| \hat{v}\left(M^{T} \xi\right) \hat{u}(\xi), \\
& \widehat{T_{u, M} v}\left(M^{T} \xi\right)=|\operatorname{det} M|^{-1} \sum_{\gamma \in \Gamma_{M^{T}}} \hat{v}(\xi+2 \pi \gamma) \overline{\hat{u}(\xi+2 \pi \gamma)}^{T},
\end{aligned}
$$

where $\Gamma_{M^{T}}$ denotes a complete set of representatives of the distinct cosets of the quotient group $\left[\left(M^{T}\right)^{-1} \mathbb{Z}^{d}\right] / \mathbb{Z}^{d}$ with $0 \in \Gamma_{M^{T}}$.

Let $\hat{a}, \widehat{b^{1}}, \ldots, \widehat{b^{L}}$ and $\hat{\tilde{a}}, \widehat{\tilde{b}^{1}}, \ldots, \widehat{\tilde{b}^{L}}$ be $r \times r$ matrices of $2 \pi$-periodic trigonometric polynomials in $d$ variables such that (1.9) is satisfied. We now discuss a discrete multiframelet transform, which consists of two parts: the multiframelet decomposition transform and the multiframelet reconstruction transform. For an input data $v^{j}: \mathbb{Z}^{d} \mapsto \mathbb{C}^{1 \times r}$ at a given fine scale $j$, the multiframelet decomposition transform computes the coarse-scale low-pass wavelet (or framelet) coefficients $v^{j-1}$ and the high-pass wavelet coefficients $w^{j-1, \ell}, \ell=1, \ldots, L$, from $v^{j}$, by

$$
v^{j-1}:=T_{\tilde{a}, M} v^{j} \quad \text { and } \quad w^{j-1, \ell}:=T_{\tilde{b}^{\ell}, M} v^{j}, \quad \ell=1, \ldots, L .
$$

From $v^{j-1}$ and $w^{j-1, \ell}, \ell=1, \ldots, L$, the multiframelet reconstruction transform can perfectly reconstruct the original signal $v^{j}$ by

$$
v^{j}=S_{a, M} v^{j-1}+\sum_{\ell=1}^{L} S_{b^{\ell}, M} w^{j-1, \ell} .
$$

The perfect reconstruction of the original signal $v^{j}$ by the multiframelet reconstruction transform in (1.20) is guaranteed by the condition in (1.9) and can be easily verified using (1.9) and (1.18) as follows: By (1.18) and (1.19), it follows from (1.9) that the Fourier series of $S_{a, M} v^{j-1}+\sum_{\ell=1}^{L} S_{b^{\ell}, M} w^{j-1, \ell}$ is given by

$$
\begin{aligned}
& |\operatorname{det} M| \widehat{v^{j-1}}\left(M^{T} \xi\right) \hat{a}(\xi)+|\operatorname{det} M| \sum_{\ell=1}^{L} \widehat{w^{j-1, \ell}}\left(M^{T} \xi\right) \widehat{b^{\ell}}(\xi)
\end{aligned}
$$

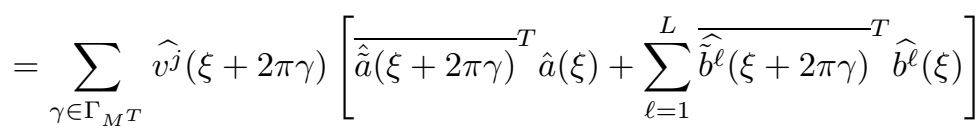

$$
\begin{aligned}
& =\sum_{\gamma \in \Gamma_{M^{T}}} \widehat{v^{j}}(\xi+2 \pi \gamma) \delta_{\gamma}=\widehat{v^{j}}(\xi) \text {. }
\end{aligned}
$$


Hence, (1.20) holds. In fact, the above argument also shows that (1.9) is a necessary and sufficient condition for the perfect reconstruction in (1.20) of the multiframelet transform. One can recursively apply the above multiframelet decomposition transform in (1.19) many times so that one obtains $v^{j-n}$ and $w^{j-n, \ell}, w^{j-(n-1), \ell}, \ldots$, $w^{j-1, \ell}, \ell=1, \ldots, L$, from which the original data $v^{j}$ can be perfectly reconstructed by the (multilevel) multiframelet reconstruction transform using (1.20).

In many applications, a datum $v$ is given as a sequence of scalar numbers, that is, $v: \mathbb{Z}^{d} \mapsto \mathbb{C}$. However, the input datum $v^{j}$ in (1.19) is a sequence of $1 \times r$ vectors, that is, $v^{j}: \mathbb{Z}^{d} \mapsto \mathbb{C}^{1 \times r}$. Thus, we have to convert a scalar sequence $v$ into a vector sequence $v^{j}$. In the following, we discuss this conversion process in detail. We say that a mapping $E: \ell\left(\mathbb{Z}^{d}\right) \mapsto\left(\ell\left(\mathbb{Z}^{d}\right)\right)^{1 \times r}$ is a vector conversion operator if $E$ is a linear operator such that $E$ is one-to-one and onto. Thus, a vector conversion operator $E$ is always invertible. In dimension one, a natural choice of a vector conversion operator $E$ is given by

$$
[E v]_{k}:=\left(v_{r k}, v_{r k+1}, \ldots, v_{r k+r-1}\right), \quad k \in \mathbb{Z}^{d}, v \in \ell\left(\mathbb{Z}^{d}\right) .
$$

Namely, one groups $r$ numbers at $r$ consecutive positions of a sequence $v$ into a $1 \times r$ vector. For a general dimension $d$, one chooses a $d \times d$ integer matrix $N$ such that $|\operatorname{det} N|=r$. Let $\Omega_{N}=\left\{\omega_{0}, \omega_{1}, \ldots, \omega_{r-1}\right\}$ denote a complete set of representatives of the distinct cosets of the quotient group $\mathbb{Z}^{d} /\left[N \mathbb{Z}^{d}\right]$. A natural choice of a vector conversion operator $E_{r}: \ell\left(\mathbb{Z}^{d}\right) \mapsto\left(\ell\left(\mathbb{Z}^{d}\right)\right)^{1 \times r}$ associated with $N$ and $\Omega_{N}$ is given by

$$
\left[E_{r} v\right]_{k}:=\left(v_{N k+\omega_{0}}, v_{N k+\omega_{1}}, \ldots, v_{N k+\omega_{r-1}}\right), \quad k \in \mathbb{Z}^{d}, v \in \ell\left(\mathbb{Z}^{d}\right) .
$$

We call $E_{r}$ in (1.22) a standard vector conversion operator. For dimension $d=1$, (1.22) becomes (1.21) if $N=r$ and $\omega_{j}=j$ for $j=0, \ldots, r-1$. For the scalar case $r=1$, one simply chooses $N=I_{d}$ and therefore, the standard vector conversion operator $E_{1}$ is the identity mapping.

Now a (multilevel) discrete multiframelet transform with a given vector conversion operator $E$ consists of the following steps:

(1) Data conversion: For a given input scalar sequence $v \in \ell\left(\mathbb{Z}^{d}\right)$, applying the given vector conversion operator $E$, one obtains a vector input sequence $v^{j}:=E v$.

(2) Decomposition: Applying the multiframelet decomposition transform in (1.19) $n$ times on the input data $v^{j}$, one obtains low-pass wavelet coefficients $v^{j-n}$ and high-pass wavelet coefficients $w^{j-n, \ell}, w^{j-(n-1), \ell}, \ldots, w^{j-1, \ell}, \ell=$ $1, \ldots, L$.

(3) Processing on wavelet coefficients: Do some required processing (such as thresholding and quantization) on the original wavelet coefficients $v^{j-n}$ and $w^{j-n, \ell}, \ldots, w^{j-1, \ell}$ to obtain after-processing coefficients $\tilde{v}^{j-n}$ and $\tilde{w}^{j-n, \ell}$, $\ldots, \tilde{w}^{j-1, \ell}$.

(4) Reconstruction: Applying the multiframelet reconstruction transform in (1.20) (replacing $v$ and $w$ by $\tilde{v}$ and $\tilde{w}$ ) $n$ times on the after-processing wavelet coefficients $\tilde{v}^{j-n}$ and $\tilde{w}^{j-n, \ell}, \ldots, \tilde{w}^{j-1, \ell}$, one obtains a reconstructed vector sequence $\tilde{v}^{j}$.

(5) Data inversion: Applying the inverse of the vector conversion operator $E$, one obtains a reconstructed scalar sequence $\tilde{v}:=E^{-1} \tilde{v}^{j}$.

If we have $\tilde{v}^{j-n}=v^{j-n}$ and $\tilde{w}^{j-k, \ell}=w^{j-k, \ell}$ (that is, no processing on wavelet coefficients), then we must have the perfect reconstruction $\tilde{v}=v$. In order to have a sparse representation of the original data $v$, it is desirable to have most high-pass 
wavelet coefficients $w^{j-n, \ell}, \ldots, w^{j-1, \ell}$ negligible. Now we describe the balancing property of a multiframelet transform.

Let $E$ be a given vector conversion operator. Let $\hat{a}, \widehat{b^{1}}, \ldots, \widehat{b^{L}}$ and $\hat{\tilde{a}}, \widehat{\tilde{b}^{1}}, \ldots, \widehat{\tilde{b}^{L}}$ be $r \times r$ matrices of $2 \pi$-periodic trigonometric polynomials in $d$ variables such that (1.9) is satisfied. In the following, we introduce the notion of balancing order from the point of view of a discrete multiframelet transform. We say that the discrete multiframelet transform in (1.19) and (1.20) has $\kappa+1$ balancing order (with respect to a given vector conversion operator $E$ ) if

(i) $T_{\tilde{a}, M} E\left(\Pi_{\kappa}\right) \subseteq E\left(\Pi_{\kappa}\right)$. In other words, for every polynomial input data $v \in \Pi_{\kappa}$, the output low-pass wavelet coefficient $T_{\tilde{a}, M} E(v)$ is still some vector polynomial sequence in $E\left(\Pi_{\kappa}\right)$ and therefore, $E^{-1} T_{\tilde{a}, M} E(v)$ is still a polynomial in $\Pi_{\kappa}$.

(ii) $T_{\tilde{b}^{\ell}, M} E\left(\Pi_{\kappa}\right)=0$ for all $\ell=1, \ldots, L$. That is, for a polynomial input data $v \in \Pi_{\kappa}$, all the output high-pass wavelet coefficients $T_{\tilde{b}^{\ell}, M} E(v)$ must vanish. In other words, there is no leakage of information from the low-frequency part to the high-frequency part.

The property $T_{\tilde{a}, M} E\left(\Pi_{\kappa}\right) \subseteq E\left(\Pi_{\kappa}\right)$ in item (i) is very important for a multilevel multiframelet transform for two reasons. First of all, this property guarantees that all the low-pass wavelet coefficients $T_{\tilde{a}, M}^{n} E(v) \in E\left(\Pi_{\kappa}\right)$ for all $n \in \mathbb{N}$ and $v \in \Pi_{\kappa}$. Consequently, after data inversion, $E^{-1} T_{\tilde{a}, M}^{n} E(v) \in \Pi_{\kappa}$ for all $v \in \Pi_{\kappa}$. Secondly, combining with item (ii), this property guarantees that all the high-pass multilevel wavelet coefficients $T_{\tilde{b}^{\ell}, M} T_{\tilde{a}, M}^{n} E(v)=0$ for all $n \in \mathbb{N}, \ell=1, \ldots, L$ and $v \in \Pi_{\kappa}$. If item (ii) holds, then we say that the high-pass filters $\tilde{b}^{\ell}, \ell=1, \ldots, L$, have $\kappa+1$ discrete vanishing moments with respect to the vector conversion operator $E$. Generally, the identity $T_{\tilde{b}^{\ell}, M} v=0$ for all $v \in\left(\Pi_{\kappa}\right)^{1 \times r}$ is not true when $r>1$. We shall present a necessary and sufficient condition in Corollary 6.1 for $T_{\tilde{b}^{\ell}, M} v=0$ for all $v \in\left(\Pi_{\kappa}\right)^{1 \times r}$.

To our best knowledge, the balancing property of a biorthogonal multiwavelet is often defined from the point of view of the function setting (see [1, 3, 4, 15, 18, [19, 20 ). Our definition of the balancing order here is not only more natural and general, but also weaker than other known related notions. We obtain a complete criterion and the essential structure of the balancing and prefiltering property of a multiframelet transform under the most general and natural condition in (1.9), since (1.9) is the necessary and sufficient condition for the perfect reconstruction of a multiframelet transform in (1.19) and (1.20). Beyond (1.9), we do not require the existence of the refinable function vectors $\phi, \tilde{\phi} \in\left(L_{2}\left(\mathbb{R}^{d}\right)\right)^{r \times 1}$ satisfying (1.11) and the vanishing moment condition in (1.14) for the wavelet function vectors $\psi^{1}, \ldots, \psi^{L}$ and $\tilde{\psi}^{1}, \ldots, \tilde{\psi}^{L}$. Our criterion is also applicable to masks satisfying (1.9) such that 1 is not an eigenvalue, or 1 is a multiple eigenvalue, of $\hat{a}(0)$ or $\hat{\tilde{a}}(0)$. Nevertheless, in sections 4 and 6 we shall present some connections of our notion of a balancing order to other related known notions of balancing order in the literature.

The structure of the paper is as follows. In section 2, we shall investigate a vector conversion operator $E$ and a closely related vector polynomial space $\mathcal{P}_{\kappa, y}$. The space $\mathcal{P}_{\kappa, y}$ will play a central role in our understanding of the balancing order of multiframelets and the approximation order of refinable function vectors. As we have seen, a multiframelet transform in (1.19) and (1.20) is built on the subdivision operator and transition operator. In order to understand the balancing order of a 
multiframelet, in section 3, we shall study several basic properties of the subdivision operator and the transition operator acting on a vector polynomial space $\mathcal{P}_{\kappa, y}$. This serves as our basis for understanding the essence of the balancing property of a multiframelet. Next in section 4 , based on the results in previous sections, we shall present the main results on the prefiltering and balancing property of a dual multiframelet and a biorthogonal multiwavelet. In this paper, we obtain a complete criterion and the essential structure for balanced dual multiframelets in the most general setting by requiring only the natural condition in (1.9). Our results in this section not only enable us to generalize some known results on a balanced biorthogonal multiwavelet to a dual multiframelet, but also provide a better picture for us to understand the prefiltering and balancing property of a multiframelet. In fact, from every given compactly supported multivariate biorthogonal multiwavelet, one can always build another (essentially equivalent) compactly supported biorthogonal multiwavelet with its balancing order matching the order of the vanishing moments of the original one. More generally, we show that if a dual multiframelet can be prefiltered, then it can be equivalently transformed into a balanced dual multiframelet with the same balancing order. We prove that every biorthogonal multiwavelet can be prefiltered to have the highest possible balancing order, while we notice that most available dual multiframelets in the literature cannot be simply prefiltered with high balancing orders and they must be designed to possess high balancing orders. In section 5 , we shall present some auxiliary results and a new canonical form of a matrix mask in high dimensions. Such a canonical form of a matrix mask greatly facilitates our investigation and constriction of multiframelets as well as many problems related to vector subdivision schemes and refinable function vectors. Finally, in section 6 we shall connect our notion and results on the balancing property of dual multiframelets in the discrete data setting with other definitions of balancing orders in the literature for orthonormal and biorthogonal multiwavelets in the function setting. Some advantages of our notion and results in this paper on balancing orders of multiframelets in the discrete data setting will be mentioned in section 6 .

\section{THE VECTOR CONVERSION OPERATOR AND THE VECTOR POLYNOMIAL SPACE}

In order to understand the balancing property of a discrete multiframelet transform, in this section we study various properties of the vector conversion operator $E$ and the vector polynomial space $\mathcal{P}_{\kappa, y}$.

To present the definition of the space $\mathcal{P}_{\kappa, y}$, let us recall some necessary notions. Let $\mathbb{N}_{0}:=\mathbb{N} \cup\{0\}$. For $x=\left(x_{1}, \ldots, x_{d}\right)^{T} \in \mathbb{R}^{d}$ and $\mu=\left(\mu_{1}, \ldots, \mu_{d}\right)^{T} \in \mathbb{N}_{0}^{d}$, we denote $\mu !:=\mu_{1} ! \cdots \mu_{d} !,|x|:=\left|x_{1}\right|+\cdots+\left|x_{d}\right|$ and $x^{\mu}:=x_{1}^{\mu_{1}} \cdots x_{d}^{\mu_{d}}$. Let $\partial_{j}$ denote the differentiation operator with respect to the $j$ th coordinate. For $\mu=\left(\mu_{1}, \ldots, \mu_{d}\right)^{T} \in \mathbb{N}_{0}^{d}, \partial:=\left(\partial_{1}, \ldots, \partial_{d}\right)^{T}$ and $\partial^{\mu}$ is the differentiation operator $\partial_{1}^{\mu_{1}} \cdots \partial_{d}^{\mu_{d}}$. Throughout this paper, we shall use the following notation:

$$
f(\xi)=g(\xi)+O\left(\|\xi\|^{\kappa+1}\right), \quad \xi \rightarrow 0
$$

to mean $\partial^{\mu} f(0)=\partial^{\mu} g(0)$ for all $|\mu| \leqslant \kappa$ and $\mu \in \mathbb{N}_{0}^{d}$.

The vanishing moments in (1.16) and the balancing order in section 1 are closely related to the notion of sum rules. For an $r \times r$ matrix $\hat{a}$ of $2 \pi$-periodic trigonometric polynomials in $d$ variables, we say that $\hat{a}$ has $\kappa+1$ sum rules (or satisfies the sum rules of order $\kappa+1$ ) with a dilation matrix $M$ (e.g., see [8, 9]) if there exists a $1 \times r$ 
vector $\hat{y}$ of $2 \pi$-periodic trigonometric polynomials in $d$ variables such that

$$
\begin{aligned}
& \hat{y}(0) \neq 0 \quad \text { and } \\
& \hat{y}\left(M^{T} \xi\right) \hat{a}(\xi+2 \pi \gamma)=\delta_{\gamma} \hat{y}(\xi)+O\left(\|\xi\|^{\kappa+1}\right), \quad \xi \rightarrow 0, \gamma \in \Gamma_{M^{T}},
\end{aligned}
$$

where $\Gamma_{M^{T}}$ denotes a complete set of representatives of the distinct cosets of the quotient group $\left[\left(M^{T}\right)^{-1} \mathbb{Z}^{d}\right] / \mathbb{Z}^{d}$ with $0 \in \Gamma_{M^{T}}$. By the Leibniz differentiation formula, it is not difficult to see that the equations in (2.2) depend only on the values $\partial^{\mu} \hat{y}(0),|\mu| \leqslant \kappa$ and $\mu \in \mathbb{N}_{0}^{d}$. See [3, 8] for more details on the definition of sum rules in the time domain.

Let $\ell_{0}\left(\mathbb{Z}^{d}\right)$ denote the linear space of all finitely supported sequences on $\mathbb{Z}^{d}$. For $y=\left\{y_{k}\right\}_{k \in \mathbb{Z}^{d}} \in\left(\ell_{0}\left(\mathbb{Z}^{d}\right)\right)^{1 \times r}$ and a positive integer $\kappa$, as in [8, 9], we define a subspace $\mathcal{P}_{\kappa, y}$ of $\left(\Pi_{\kappa}\right)^{1 \times r}$ by

$$
\mathcal{P}_{\kappa, y}:=\left\{h * y: h \in \Pi_{\kappa}\right\},
$$

where

$$
h * y:=\sum_{k \in \mathbb{Z}^{d}} h(\cdot-k) y_{k}, \quad h \in \Pi_{\kappa} .
$$

Denote $\Pi:=\bigcup_{\kappa=0}^{\infty} \Pi_{\kappa}$, the set of all polynomials in $d$ variables. For a vector polynomial $g \in \Pi^{1 \times r}$, it is evident that the vector polynomial $g$ is uniquely determined by its restriction $\left.g\right|_{\mathbb{Z}^{d}}$ on the integer lattice $\mathbb{Z}^{d}$. Therefore, throughout the paper, $g \in \Pi^{1 \times r}$ means either a vector polynomial with domain $\mathbb{R}^{d}$ or a vector polynomial sequence $\left.g\right|_{\mathbb{Z}^{d}}$ with domain $\mathbb{Z}^{d}$, which can be easily distinguished from the context.

The following result shows that the space $\mathcal{P}_{\kappa, y}$ only depends on the values $\partial^{\mu} \hat{y}(0)$, $|\mu| \leqslant \kappa$.

Proposition 2.1. Let $y: \mathbb{Z}^{d} \mapsto \mathbb{C}^{1 \times r}$ be a finitely supported sequence on $\mathbb{Z}^{d}$. Then

$$
\begin{aligned}
h * y & =[h(\cdot-i \partial) \hat{y}](0)=\sum_{\mu \in \mathbb{N}_{0}^{d}}\left(\partial^{\mu} h\right)(\cdot) \frac{(-i \partial)^{\mu}}{\mu !} \hat{y}(0) \\
& =\sum_{\mu \in \mathbb{N}_{0}^{d}}\left[\left(\partial^{\mu} h\right)(-i \partial) \hat{y}\right](0) \frac{(\cdot)^{\mu}}{\mu !}, \quad h \in \Pi
\end{aligned}
$$

and for $h \in \Pi$,

$$
\begin{aligned}
& \partial^{\nu}(h * y)=\left(\partial^{\nu} h\right) * y, \quad \nu \in \mathbb{N}_{0}^{d} \quad \text { and } \\
& (h * y)(\cdot-k)=h(\cdot-k) * y, \quad k \in \mathbb{R}^{d} .
\end{aligned}
$$

Consequently, for any $\kappa \in \mathbb{N}_{0}$, the space $\mathcal{P}_{\kappa, y}$ is contained in $\left(\Pi_{\kappa}\right)^{1 \times r}$ and is invariant under both differentiation and shifts in $\mathbb{R}^{d}$. If $\hat{y}(0) \neq 0$, then $\operatorname{dim}\left(\mathcal{P}_{\kappa, y}\right)=$ $\operatorname{dim}\left(\Pi_{\kappa}\right)$.

Proof. By the definition of $h * y$ in (2.4), we have $[h * y]_{j}=\sum_{k \in \mathbb{Z}^{d}} h(j-k) y_{k}$. Since $h \in \Pi$ is a polynomial, using the Taylor expansion of $h$ at the point $j$, we deduce that

$$
h(j-k)=\sum_{\mu \in \mathbb{N}_{0}^{d}}\left(\partial^{\mu} h\right)(j) \frac{(-k)^{\mu}}{\mu !}, \quad h \in \Pi .
$$

Consequently,

$$
[h * y]_{j}=\sum_{k \in \mathbb{Z}^{d}} h(j-k) y_{k}=\sum_{\mu \in \mathbb{N}_{0}^{d}}\left(\partial^{\mu} h\right)(j) \sum_{k \in \mathbb{Z}^{d}} y_{k} \frac{(-k)^{\mu}}{\mu !}, \quad h \in \Pi .
$$


On the other hand, by $\hat{y}(\xi)=\sum_{k \in \mathbb{Z}^{d}} y_{k} e^{-i k \cdot \xi}$, we observe that $\frac{(-i \partial)^{\mu}}{\mu !} \hat{y}(0)=$ $\sum_{k \in \mathbb{Z}^{d}} y_{k} \frac{(-k)^{\mu}}{\mu !}$. Now from (2.7), it is straightforward to see that (2.5) holds, since by the Taylor expansion of $h$, we have

$$
\begin{aligned}
& h(x-i \partial)=\sum_{\mu \in \mathbb{N}_{0}^{d}}\left(\partial^{\mu} h\right)(x) \frac{(-i \partial)^{\mu}}{\mu !} \text { and } \\
& h(x-i \partial)=\sum_{\mu \in \mathbb{N}_{0}^{d}} \frac{x^{\mu}}{\mu !}\left(\partial^{\mu} h\right)(-i \partial), \quad x \in \mathbb{R}^{d}, h \in \Pi .
\end{aligned}
$$

Note that $\partial$ here only acts on the frequency variable $\xi$, not on the time variable $x$.

By (2.5), we see that $\partial^{\nu}(h * y)=\left(\partial^{\nu} h\right) * y$ for all $\nu \in \mathbb{N}_{0}^{d}$ and $h \in \Pi$. Therefore, $\mathcal{P}_{\kappa, y}$ is invariant under differentiation. Similarly, by (2.5), we have $(h * y)(\cdot-k)=$ $h(\cdot-k) * y$ for all $k \in \mathbb{R}^{d}$ and $h \in \Pi$. So, $\mathcal{P}_{\kappa, y}$ is invariant under shifts in $\mathbb{R}^{d}$.

If $\hat{y}(0) \neq 0$, then it is easy to verify that the mapping $h \in \Pi_{\kappa} \mapsto h * y \in \mathcal{P}_{\kappa, y}$ is one-to-one, since (2.5) and $h * y=0$ will force $h=0$. Consequently, $\operatorname{dim}\left(\mathcal{P}_{\kappa, y}\right)=$ $\operatorname{dim}\left(\Pi_{\kappa}\right)$ if $\hat{y}(0) \neq 0$.

Due to Proposition 2.1, as long as $\hat{y}(\xi)$ is $\kappa$ th differentiable at $\xi=0$, then we can always define a space $\mathcal{P}_{\kappa, y}:=\left\{h * y: h \in \Pi_{\kappa}\right\}$ using the definition of $h * y$ in (2.5) instead of (2.4). In other words, in order to define the space $\mathcal{P}_{\kappa, y}$, it is not necessary to require that $\hat{y}$ be a $1 \times r$ vector of $2 \pi$-periodic trigonometric polynomials.

The following result connects the vector conversion operator $E_{r}$ in (1.22) to a vector polynomial subspace $\mathcal{P}_{\kappa, y}$.

Proposition 2.2. Let $N$ be a $d \times d$ real-valued invertible matrix and let $\omega_{0}, \ldots, \omega_{r-1}$ $\in \mathbb{R}^{d}$. Let $y \in\left(\ell_{0}\left(\mathbb{Z}^{d}\right)\right)^{1 \times r}$ be a finitely supported sequence satisfying

$$
\frac{(-i \partial)^{\mu}}{\mu !} \hat{y}(0)=\left(\frac{\left(N^{-1} \omega_{0}\right)^{\mu}}{\mu !}, \ldots, \frac{\left(N^{-1} \omega_{r-1}\right)^{\mu}}{\mu !}\right), \quad|\mu| \leqslant \kappa, \mu \in \mathbb{N}_{0}^{d} .
$$

In other words,

$$
\begin{aligned}
& \hat{y}(\xi)=\hat{Y}(\xi)+O\left(\|\xi\|^{\kappa+1}\right), \quad \xi \rightarrow 0 \\
& \text { with } \hat{Y}(\xi):=\left(e^{i N^{-1} \omega_{0} \cdot \xi}, e^{i N^{-1} \omega_{1} \cdot \xi}, \ldots, e^{i N^{-1} \omega_{r-1} \cdot \xi}\right) .
\end{aligned}
$$

Then for any nonnegative integer $\kappa$,

$$
\begin{aligned}
E_{r}(h) & =h(N \cdot) * Y=T_{I_{r} \delta, N}\left(h * Y^{N}\right) \\
& =\left(h\left(N \cdot+\omega_{0}\right), \ldots, h\left(N \cdot+\omega_{r-1}\right)\right), \quad h \in \Pi_{\kappa},
\end{aligned}
$$

where $\widehat{Y^{N}}(\xi):=\hat{Y}\left(N^{T} \xi\right)=\left(e^{i \omega_{0} \cdot \xi}, e^{i \omega_{1} \cdot \xi}, \ldots, e^{i \omega_{r-1} \cdot \xi}\right)$. Consequently,

$$
\begin{gathered}
\left\{\left(h\left(N \cdot+\omega_{0}\right), h\left(N \cdot+\omega_{1}\right), \ldots, h\left(N \cdot+\omega_{r-1}\right)\right): h \in \Pi_{\kappa}\right\} \\
=\left\{h * y: h \in \Pi_{\kappa}\right\}=\mathcal{P}_{\kappa, y} .
\end{gathered}
$$

In particular, for the vector conversion operator $E_{r}$ in (1.22), we have $E_{r}\left(\Pi_{\kappa}\right)=$ $\mathcal{P}_{\kappa, Y}$ for every $\kappa \in \mathbb{N}_{0}$, where $\hat{Y}$ is defined in (2.9).

Proof. Since $N$ is invertible, it is easy to see that the set on the left side of (2.11) agrees with

$$
\left\{\left(h\left(\cdot+N^{-1} \omega_{0}\right), h\left(\cdot+N^{-1} \omega_{1}\right), \ldots, h\left(\cdot+N^{-1} \omega_{r-1}\right)\right): h \in \Pi_{\kappa}\right\} .
$$


Therefore, using a Taylor expansion, for $h \in \Pi_{\kappa}$, we have

$$
h\left(\cdot+N^{-1} \omega_{j}\right)=\sum_{\mu \in \mathbb{N}_{0}^{d}}\left(\partial^{\mu} h\right)(\cdot) \frac{\left(N^{-1} \omega_{j}\right)^{\mu}}{\mu !}=\sum_{|\mu| \leqslant \kappa}\left(\partial^{\mu} h\right)(\cdot) \frac{\left(N^{-1} \omega_{j}\right)^{\mu}}{\mu !} .
$$

On the other hand, for any sequence $y$ satisfying (2.8), by (2.5) or [9, (2.13)], for $h \in \Pi_{\kappa}$, we deduce that

$$
\begin{aligned}
h * y & =\sum_{\mu \in \mathbb{N}_{0}^{d}}\left(\partial^{\mu} h\right)(\cdot) \frac{(-i \partial)^{\mu}}{\mu !} \hat{y}(0)=\sum_{|\mu| \leqslant \kappa}\left(\partial^{\mu} h\right)(\cdot)\left(\frac{\left(N^{-1} \omega_{0}\right)^{\mu}}{\mu !}, \ldots, \frac{\left(N^{-1} \omega_{r-1}\right)^{\mu}}{\mu !}\right) \\
& =\left(h\left(\cdot+N^{-1} \omega_{0}\right), \ldots, h\left(\cdot+N^{-1} \omega_{r-1}\right)\right) .
\end{aligned}
$$

By (3.1), we have $h(N \cdot) * Y=T_{I_{r} \delta, N}\left(h * Y^{N}\right)$. Consequently, we conclude that (2.11) holds.

We shall see that the converse direction of Proposition 2.2 is also true. In order to do so, we need an auxiliary result. For a square matrix $\hat{U}(\xi)$ of $2 \pi$-periodic trigonometric polynomials in $d$ variables, we say that $\hat{U}$ is strongly invertible if $\hat{U}(\xi)$ is invertible for all $\xi \in \mathbb{R}^{d}$ and the inverse of $\hat{U}(\xi)$ is also a matrix of $2 \pi$-periodic trigonometric polynomials in $d$ variables.

In order to understand the space $\mathcal{P}_{\kappa, y}$ better and to prove the converse direction of Proposition 2.2, we need the following technical result, whose proof will be given in section 5 .

Lemma 2.3. Let $\hat{y}$ and $\hat{\tilde{y}}$ be two $s \times r(s<r)$ vectors of functions that are infinitely differentiable at the origin. Suppose that $\hat{y}(0)$ and $\hat{\tilde{y}}(0)$ have the full rank $s$. If $r>1$, then for any nonnegative integer $n$, there is a strongly invertible $r \times r$ matrix $\hat{U}(\xi)$ of $2 \pi$-periodic trigonometric polynomials such that

$$
\partial^{\mu} \hat{y}(0)=\partial^{\mu}[\hat{\tilde{y}}(\cdot) \hat{U}(\cdot)](0), \quad|\mu|<n, \mu \in \mathbb{N}_{0}^{d},
$$

or equivalently, $\hat{y}(\xi)=\hat{\tilde{y}}(\xi) \hat{U}(\xi)+O\left(\|\xi\|^{n}\right), \xi \rightarrow 0$.

Conversely, for any $y \in\left(\ell_{0}\left(\mathbb{Z}^{d}\right)\right)^{1 \times r}$ with $\hat{y}(0) \neq 0$ and for any nonnegative integer $\kappa$, we show that there always exists a vector conversion operator $E$ such that $E\left(\Pi_{\kappa}\right)=\mathcal{P}_{\kappa, y}$.

Theorem 2.4. Let $\kappa \in \mathbb{N}_{0}$ and $y \in\left(\ell_{0}\left(\mathbb{Z}^{d}\right)\right)^{1 \times r}$ with $\hat{y}(0) \neq 0$. Then there exists a strongly invertible $r \times r$ matrix $\hat{U}(\xi)=\sum_{k \in \mathbb{Z}^{d}} U_{k} e^{-i k \cdot \xi}$ of $2 \pi$-periodic trigonometric polynomials in d variables such that the following vector conversion operator

$$
E=C_{U} \circ E_{r}: \ell\left(\mathbb{Z}^{d}\right) \stackrel{E_{r}}{\longmapsto}\left(\ell\left(\mathbb{Z}^{d}\right)\right)^{1 \times r} \stackrel{C_{U}}{\longmapsto}\left(\ell\left(\mathbb{Z}^{d}\right)\right)^{1 \times r}
$$

satisfies $E\left(\Pi_{\kappa}\right)=\mathcal{P}_{\kappa, y}$, where $E_{r}$ is the standard vector conversion operator in (1.22) and the convolution operator $C_{U}$ is defined to be

$$
C_{U} w:=w * U:=\sum_{k \in \mathbb{Z}^{d}} w \cdot-k U_{k}
$$

for $w \in\left(\ell\left(\mathbb{Z}^{d}\right)\right)^{1 \times r}$. Moreover,

$$
E(h)=h(N \cdot) * y=T_{I_{r} \delta, N}\left(h * y^{N}\right), \quad h \in \Pi_{\kappa} \quad \text { with } \quad \widehat{y^{N}}(\xi):=\hat{y}\left(N^{T} \xi\right) .
$$


Proof. If $r=1$, then the claim holds with $\hat{U}=1$. Thus, we assume $r>1$. Let $\hat{Y}$ be defined in (2.9). By Proposition 2.2, we have $E_{r}\left(\Pi_{\kappa}\right)=\mathcal{P}_{\kappa, Y}$. Since $\hat{Y}(0) \neq 0$ and $\hat{y}(0) \neq 0$, by Lemma 2.3 , there is a strongly invertible matrix $\hat{U}$ such that

$$
\hat{y}(\xi)=\hat{Y}(\xi) \hat{U}(\xi)+O\left(\|\xi\|^{\kappa+1}\right), \quad \xi \rightarrow 0 .
$$

Therefore, for $h \in \Pi_{\kappa}$, by (2.10), we have

$E(h)=C_{U}\left(E_{r}(h)\right)=C_{U}(h(N \cdot) * Y)=h(N \cdot) * Y * U=h(N \cdot) * y=T_{I_{r} \delta, N}\left(h * y^{N}\right)$

and

$$
\begin{aligned}
E\left(\Pi_{\kappa}\right) & =C_{U}\left(E_{r}\left(\Pi_{\kappa}\right)\right)=C_{U}\left(\mathcal{P}_{\kappa, Y}\right)=\left\{(h * Y) * U: h \in \Pi_{\kappa}\right\} \\
& =\left\{h *(Y * U): h \in \Pi_{\kappa}\right\}=\mathcal{P}_{\kappa, y} .
\end{aligned}
$$

That is, we have $E\left(\Pi_{\kappa}\right)=\mathcal{P}_{\kappa, y}$.

In order to show that $E$ is a vector conversion operator, since $E_{r}$ is a standard vector conversion operator, it suffices to show that $C_{U}$ is one-to-one and onto. Denote $\hat{V}(\xi)=\sum_{k \in \mathbb{Z}^{d}} V_{k} e^{-i k \cdot \xi}:=\hat{U}(\xi)^{-1}$. Since $\hat{U}$ is strongly invertible, $\hat{V}$ is a matrix of $2 \pi$-periodic trigonometric polynomials. By $\hat{U}(\xi) \hat{V}(\xi)=I_{r}$, we deduce that $\sum_{k \in \mathbb{Z}^{d}} U_{j-k} V_{k}=\delta_{j} I_{r}$ for all $j \in \mathbb{Z}^{d}$. Now we verify that

$$
C_{V}\left(C_{U} w\right)=\left(C_{U} w\right) * V=(w * U) * V=w *(U * V)=w \quad \forall w \in\left(\ell\left(\mathbb{Z}^{d}\right)\right)^{1 \times r} .
$$

In fact, by calculation, for $j \in \mathbb{Z}^{d}$, we have

$$
\begin{aligned}
{\left[\left(C_{U} w\right) * V\right]_{j} } & =\sum_{k \in \mathbb{Z}^{d}}\left[C_{U} w\right]_{j-k} V_{k}=\sum_{k \in \mathbb{Z}^{d}} w_{j-k-n} U_{n} V_{k} \\
& =\sum_{n \in \mathbb{Z}^{d}} w_{j-n} \sum_{k \in \mathbb{Z}^{d}} U_{n-k} V_{k}=\sum_{n \in \mathbb{Z}^{d}} w_{j-n} \delta_{n} I_{r}=w_{j} .
\end{aligned}
$$

Hence, (2.16) is verified and consequently, $C_{U}$ is one-to-one and onto.

To further study the vector polynomial space $\mathcal{P}_{\kappa, y}$, we need the following simple fact later.

Lemma 2.5. The following statements hold.

(1) For any given complex numbers $c_{\mu},|\mu| \leqslant \kappa$ and $\mu \in \mathbb{N}_{0}^{d}$, there is a $2 \pi$ periodic trigonometric polynomial $\hat{c}$ such that $\partial^{\mu} \hat{c}(0)=c_{\mu}$ for all $|\mu| \leqslant \kappa$.

(2) For a $d \times d$ real-valued matrix $N$ and a $2 \pi$-periodic trigonometric polynomial $\widehat{c^{1}}$, there is a $2 \pi$-periodic trigonometric polynomial $\widehat{c^{2}}$ such that

$$
\widehat{c^{2}}(\xi)=\widehat{c^{1}}(N \xi)+O\left(\|\xi\|^{\kappa+1}\right), \quad \xi \rightarrow 0 .
$$

(3) Let $M$ be a $d \times d$ dilation matrix. For a $2 \pi$-periodic trigonometric polynomial $\hat{c}$ with $\hat{c}(0) \neq 0$, there exist $2 \pi$-periodic trigonometric polynomials $\widehat{c^{1}}$ and $\widehat{c^{2}}$ such that $\widehat{c^{1}}(0)=\widehat{c^{2}}(0)=1$ and

$$
\hat{c}(\xi)=\hat{c}(0) \frac{\widehat{c^{1}}\left(M^{T} \xi\right)}{\widehat{c^{1}}(\xi)}+O\left(\|\xi\|^{\kappa+1}\right)=\hat{c}(0) \frac{\widehat{c^{2}}(\xi)}{\widehat{c}^{2}\left(M^{T} \xi\right)}+O\left(\|\xi\|^{\kappa+1}\right), \quad \xi \rightarrow 0 .
$$

Proof. It is straightforward to prove items (1) and (2). To prove item (3), since $\hat{c}(0) \neq 0$ and $\hat{c}$ is a $2 \pi$-periodic trigonometric polynomial, it is well known that $\hat{\phi}(\xi):=\prod_{j=1}^{\infty}\left[\hat{c}\left(\left(M^{T}\right)^{-j} \xi\right) / \hat{c}(0)\right]$ is a well-defined $C^{\infty}$ function with $\hat{\phi}(0)=1$. Choose $\widehat{c^{1}}$ and $\widehat{c^{2}}$ to be $2 \pi$-periodic trigonometric polynomials such that $\widehat{c^{1}}(\xi)=$ 
$\hat{\phi}(\xi)+O\left(\|\xi\|^{\kappa+1}\right)$ and $\widehat{c^{2}}(\xi)=1 / \hat{\phi}(\xi)+O\left(\|\xi\|^{\kappa+1}\right)$ as $\xi \rightarrow 0$. It is evident that (2.17) holds.

The following result is implicitly given in [9, Lemma 3.3] and will be used frequently in this paper. For the convenience of the reader, we present a complete proof here.

Lemma 2.6. Let $N$ be a $d \times d$ invertible real-valued matrix. Let $y, \stackrel{\circ}{y}: \mathbb{Z}^{d} \mapsto \mathbb{C}^{1 \times r}$ be two finitely supported sequences on $\mathbb{Z}^{d}$ with $\hat{y}(0) \neq 0$. Then $\mathcal{P}_{\kappa, \dot{y}} \subseteq \mathcal{P}_{\kappa, y}$ if and only if there exists a $2 \pi$-periodic trigonometric polynomial $\hat{c}$ such that

$$
\hat{\hat{y}}(\xi)=\hat{c}(N \xi) \hat{y}(\xi)+O\left(\|\xi\|^{\kappa+1}\right), \quad \xi \rightarrow 0 .
$$

In particular, $\mathcal{P}_{\kappa, \grave{y}}=\mathcal{P}_{\kappa, y}$ if and only if (2.18) holds with $\hat{c}(0) \neq 0$.

Proof. By item (2) of Lemma 2.5, it suffices to prove the claim for $N=I_{d}$. So, in the following, we assume $N=I_{d}$. By Proposition 2.1. we see that $h * c \in \Pi_{\kappa}$ for all $h \in \Pi_{\kappa}$. Moreover, we have $\left\{h * c: h \in \Pi_{\kappa}\right\}=\Pi_{\kappa}$ if and only if $\hat{c}(0) \neq 0$. If (2.18) holds, we conclude that

$$
\mathcal{P}_{\kappa, \grave{y}}=\left\{h * c * y: h \in \Pi_{\kappa}\right\} \subseteq\left\{h * y: h \in \Pi_{\kappa}\right\}=\mathcal{P}_{\kappa, y} .
$$

Now we prove the converse. If $r=1$, then by $\hat{y}(0) \neq 0$, we have $\mathcal{P}_{\kappa, y}=\Pi_{\kappa}$ and we can simply take a $2 \pi$-periodic trigonometric polynomial $\hat{c}$ such that $\hat{c}(\xi)=$ $\hat{y}(\xi) / \hat{y}(\xi)+O\left(\|\xi\|^{\kappa+1}\right)$ as $\xi \rightarrow 0$. Now all the claims follow easily for the scalar case $r=1$.

Suppose $r>1$. By Lemma 2.3, there is a strongly invertible $r \times r$ matrix $\hat{U}(\xi)$ of $2 \pi$-periodic trigonometric polynomials such that

$$
\hat{y}(\xi) \hat{U}(\xi)=(1,0, \ldots, 0)+O\left(\|\xi\|^{\kappa+1}\right), \quad \xi \rightarrow 0 .
$$

Since $\hat{U}$ is strongly invertible, the linear mapping $\left(\ell\left(\mathbb{Z}^{d}\right)\right)^{1 \times r} \mapsto\left(\ell\left(\mathbb{Z}^{d}\right)\right)^{1 \times r}$ defined by $u \mapsto u * U$ is one-to-one and onto (see the proof of Theorem 2.4). Now it is easy to see that $\mathcal{P}_{\kappa, \tilde{y}} \subseteq \mathcal{P}_{\kappa, y}$ if and only if $\mathcal{P}_{\kappa, \tilde{y}} \subseteq \mathcal{P}_{\kappa, \tilde{y}}$, where $\hat{\hat{y}}(\xi):=\hat{\hat{y}}(\xi) \hat{U}(\xi)$ and $\hat{\tilde{y}}(\xi):=\hat{y}(\xi) \hat{U}(\xi)$. By (2.19) and Proposition 2.1. we deduce that

$$
\mathcal{P}_{\kappa, \tilde{y}}=\left\{h * \tilde{y}: h \in \Pi_{\kappa}\right\}=\left\{(h, 0, \ldots, 0): h \in \Pi_{\kappa}\right\} .
$$

By Proposition 2.1 again, we see that $\mathcal{P}_{\kappa, \tilde{y}}$ is a subspace of $\left(\Pi_{\kappa}\right)^{1 \times r}$ and $\mathcal{P}_{\kappa, \tilde{y}} \subseteq$ $\mathcal{P}_{\kappa, \tilde{y}}=\left\{[h, 0, \ldots, 0]: h \in \Pi_{\kappa}\right\}$ if and only if

$\hat{\tilde{y}}_{\ell}(\xi)=O\left(\|\xi\|^{\kappa+1}\right), \quad \xi \rightarrow 0, \ell=2, \ldots, r \quad$ with $\quad\left(\hat{\tilde{y}}_{1}(\xi), \hat{\tilde{y}}_{2}(\xi), \ldots, \hat{\tilde{y}}_{r}(\xi)\right):=\hat{\hat{\tilde{y}}}(\xi)$.

Take $\hat{c}(\xi)=\hat{\hat{\tilde{y}}}_{1}(\xi)$. Then it follows from the above relations that $\hat{\tilde{y}}(\xi)=\hat{c}(\xi) \hat{\tilde{y}}(\xi)+$ $O\left(\|\xi\|^{\kappa+1}\right)$ as $\xi \rightarrow 0$. Since $\hat{U}$ is strongly invertible, we conclude that (2.18) holds with $N=I_{d}$.

In the next few sections, we shall see that the balancing order of a multiframelet transform is closely related to the vector conversion operator $E$ and the vector polynomial subspace $\mathcal{P}_{\kappa, y}$. We shall see later that in order to have the balancing property of a multiframelet transform, it is very natural to require $E\left(\Pi_{\kappa}\right)=\mathcal{P}_{\kappa, y}$. For the scalar case $r=1$, by $\hat{y}(0) \neq 0$ and (2.5), it is easy to see that $\mathcal{P}_{\kappa, y}=\Pi_{\kappa}$. Therefore, regardless of the choice of the sequence $y, E\left(\Pi_{\kappa}\right)=\mathcal{P}_{\kappa, y}$ is always true for the identity conversion operator $E$. However, for multiplicity $r>1, \mathcal{P}_{\kappa, y}$ has the same dimension as the linear space $\Pi_{\kappa}$ and therefore, it is a proper subspace of 
the vector polynomial space $\left(\Pi_{\kappa}\right)^{1 \times r}$. The vector polynomial space $\mathcal{P}_{\kappa, y}$ also plays a critical role in the study of the approximation properties of refinable function vectors and sum rules of matrix masks; see [8, 9] for more details on the vector polynomial space $\mathcal{P}_{\kappa, y}$.

\section{Some Properties of THE SUbDivision OPERATORS AND TRANSITION OPERATORS}

In this section, we shall study various properties of a subdivision operator $S_{u, M}$ and a transition operator $T_{u, M}$ in (1.17) acting on a general vector polynomial space $\mathcal{P}_{\kappa, y}$.

Since the balancing property of a multiframelet transform is closely related to the action of the subdivision operator and the transition operator on the space $E\left(\Pi_{\kappa}\right)$, by the connection of the spaces $E\left(\Pi_{\kappa}\right)$ and $\mathcal{P}_{\kappa, y}$ in Proposition 2.2 and Theorem 2.4. it is of interest and importance to study the properties of the subdivision operator and transition operator acting on the space $\mathcal{P}_{\kappa, y}$. We address this issue here. The results in this section will serve as our basis for understanding the notion of sum rules, (discrete) vanishing moments, and balancing orders of a multiframelet transform.

For a transition operator $T_{u, M}$ acting on a space $\mathcal{P}_{\kappa, y}$, following [11, we have the following result.

Proposition 3.1. Let $u: \mathbb{Z}^{d} \mapsto \mathbb{C}^{r \times r}$ be a finitely supported sequence of $r \times r$ matrices on $\mathbb{Z}^{d}$. Let $y \in\left(\ell_{0}\left(\mathbb{Z}^{d}\right)\right)^{1 \times r}$ be a finitely supported sequence of $1 \times r$ vectors on $\mathbb{Z}^{d}$. Then for any nonnegative integer $\kappa$,

$$
T_{u, M}(h * y)=h(M \cdot) * \stackrel{\circ}{y}, \quad h \in \Pi_{\kappa},
$$

where $\dot{y}$ is a finitely supported sequence of $1 \times r$ vectors on $\mathbb{Z}^{d}$ such that

$$
\hat{y}\left(M^{T} \xi\right)=\hat{y}(\xi) \overline{\hat{u}(\xi)}^{T}+O\left(\|\xi\|^{\kappa+1}\right), \quad \xi \rightarrow 0 .
$$

As a consequence of (3.1), we have

(1) $T_{u, M} \mathcal{P}_{\kappa, y}=\mathcal{P}_{\kappa, \grave{y}}$ with $\grave{y}$ satisfying (3.2). If in addition $\hat{y}(0) \overline{\hat{u}(0)}^{T} \neq 0$, then the restricted mapping $\left.T_{u, M}\right|_{\mathcal{P}_{\kappa, y}}: \mathcal{P}_{\kappa, y} \mapsto \mathcal{P}_{\kappa, \grave{y}}$ is one-to-one and onto.

(2) $T_{u, M}(h * y)=0$ for all $h \in \Pi_{\kappa}$ if and only if $\hat{y}(\xi) \overline{\hat{u}(\xi)}^{T}=O\left(\|\xi\|^{\kappa+1}\right)$ as $\xi \rightarrow 0$.

(3) For $y \in\left(\ell_{0}\left(\mathbb{Z}^{d}\right)\right)^{1 \times r}$ with $\hat{y}(0) \neq 0, T_{u, M} \mathcal{P}_{\kappa, y} \subseteq \mathcal{P}_{\kappa, y}$ if and only if there exists a $2 \pi$-periodic trigonometric polynomial $\hat{c}$ such that

$$
\hat{y}(\xi) \overline{\hat{u}(\xi)}^{T}=\hat{c}(\xi) \hat{y}\left(M^{T} \xi\right)+O\left(\|\xi\|^{\kappa+1}\right), \quad \xi \rightarrow 0 .
$$

Moreover, $T_{u, M} \mathcal{P}_{\kappa, y}=\mathcal{P}_{\kappa, y}$ if and only if (3.3) holds with $\hat{c}(0) \neq 0$.

Proof. By the definition of the transition operator $T_{u, M}$ in (1.17), for $h \in \Pi_{\kappa}$, we have

$$
\begin{aligned}
{\left[T_{u, M}(h * y)\right]_{j} } & =\sum_{k \in \mathbb{Z}^{d}}(h * y)_{k}{\overline{u_{k-M j}}}^{T}=\sum_{k \in \mathbb{Z}^{d}} \sum_{n \in \mathbb{Z}^{d}} h(k-n) y_{n}{\overline{u_{k-M j}}}^{T} \\
& =\sum_{k \in \mathbb{Z}^{d}} \sum_{n \in \mathbb{Z}^{d}} h(M j+k) y_{n}{\overline{u_{k+n}}}^{T} .
\end{aligned}
$$


By the Taylor expansion of $h(M \cdot)$ at the point $j$, we have

$$
h(M j+k)=h\left(M\left(j+M^{-1} k\right)\right)=\sum_{\mu \in \mathbb{N}_{0}^{d}}\left[\partial^{\mu}(h(M \cdot))\right](j) \frac{\left(M^{-1} k\right)^{\mu}}{\mu !} .
$$

Hence, we have

$$
\left[T_{u, M}(h * y)\right]_{j}=\sum_{|\mu| \leqslant \kappa}\left[\partial^{\mu}(h(M \cdot))\right](j) \sum_{k \in \mathbb{Z}^{d}} \sum_{n \in \mathbb{Z}^{d}} y_{n}{\overline{u_{k+n}}}^{T} \frac{\left(M^{-1} k\right)^{\mu}}{\mu !}, \quad h \in \Pi_{\kappa} .
$$

Denote $\hat{v}(\xi):=\hat{y}(\xi) \overline{\hat{u}(\xi)}^{T}$. By the assumption that $\hat{\hat{y}}\left(M^{T} \xi\right)=\hat{y}(\xi) \overline{\hat{u}}(\xi)^{T}+O\left(\|\xi\|^{\kappa+1}\right)$ as $\xi \rightarrow 0$, we have

$$
\hat{y}(\xi)=\hat{y}\left(\left(M^{T}\right)^{-1} \xi\right){\overline{\hat{u}\left(\left(M^{T}\right)^{-1} \xi\right)}}^{T}+O\left(\|\xi\|^{\kappa+1}\right)=\hat{v}\left(\left(M^{T}\right)^{-1} \xi\right)+O\left(\|\xi\|^{\kappa+1}\right), \xi \rightarrow 0 .
$$

That is,

$$
\begin{aligned}
\sum_{k \in \mathbb{Z}^{d}} \stackrel{\circ}{y}_{k} e^{-i k \cdot \xi} & =\sum_{k \in \mathbb{Z}^{d}} v_{k} e^{-i k \cdot\left(M^{T}\right)^{-1} \xi}+O\left(\|\xi\|^{\kappa+1}\right) \\
& =\sum_{k \in \mathbb{Z}^{d}} v_{k} e^{-i M^{-1} k \cdot \xi}+O\left(\|\xi\|^{\kappa+1}\right), \quad \xi \rightarrow 0 .
\end{aligned}
$$

Now for $|\mu| \leqslant \kappa$, we deduce from the above relation that

$$
\begin{aligned}
\frac{(-i \partial)^{\mu}}{\mu !} \hat{y}(0) & =\left.\left[\frac{(-i \partial)^{\mu}}{\mu !} \sum_{k \in \mathbb{Z}^{d}} \stackrel{\circ}{y}_{k} e^{-i k \cdot \xi}\right]\right|_{\xi=0} \\
& =\left.\left[\frac{(-i \partial)^{\mu}}{\mu !} \sum_{k \in \mathbb{Z}^{d}} v_{k} e^{-i M^{-1} k \cdot \xi}\right]\right|_{\xi=0}=\sum_{k \in \mathbb{Z}^{d}} v_{k} \frac{\left(-M^{-1} k\right)^{\mu}}{\mu !} .
\end{aligned}
$$

Since $\hat{v}(\xi)=\hat{y}(\xi) \overline{\hat{u}(\xi)}^{T}$, we have $v_{k}=\sum_{n \in \mathbb{Z}^{d}} y_{n}{\overline{u_{n-k}}}^{T}$. Hence,

$$
\begin{aligned}
\frac{(-i \partial)^{\mu}}{\mu !} \hat{y}(0) & =\sum_{k \in \mathbb{Z}^{d}} v_{k} \frac{\left(-M^{-1} k\right)^{\mu}}{\mu !}=\sum_{k \in \mathbb{Z}^{d}} v_{-k} \frac{\left(M^{-1} k\right)^{\mu}}{\mu !} \\
& =\sum_{k \in \mathbb{Z}^{d}} \sum_{n \in \mathbb{Z}^{d}} y_{n} \bar{u}_{n+k}^{T} \frac{\left(M^{-1} k\right)^{\mu}}{\mu !} .
\end{aligned}
$$

By (3.4) and (2.5), we conclude that

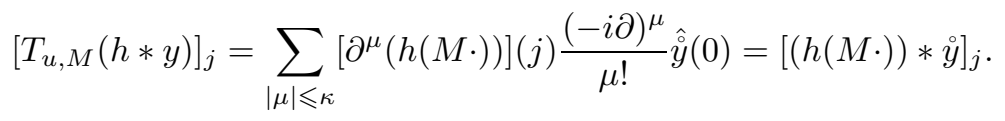

So, (3.1) has been verified.

By (3.1), it is evident that item (1) holds. If $\left.\hat{y}(0)=\hat{y}(0) \overline{\hat{u}}^{T}\right)^{T} \neq 0$, by Proposition 2.1] then $\operatorname{dim}\left(\mathcal{P}_{\kappa, y}\right)=\operatorname{dim}\left(\mathcal{P}_{\kappa, \grave{y}}\right)=\operatorname{dim}\left(\Pi_{\kappa}\right)$. Since $T_{u, M} \mathcal{P}_{\kappa, y}=\mathcal{P}_{\kappa, \grave{y}}$, the restricted mapping $\left.T_{u, M}\right|_{\mathcal{P}_{\kappa, y}}: \mathcal{P}_{\kappa, y} \mapsto \mathcal{P}_{\kappa, \check{y}}$ must be one-to-one and onto.

For $h \in \Pi_{\kappa}$, by (3.1), we have $T_{u, M}(h * y)=h(M \cdot) * \stackrel{y}{ }$. So, $T_{u, M}(h * y)=0$ for all $h \in \Pi_{\kappa}$ if and only if

$$
\sum_{|\mu| \leqslant \kappa}\left[\partial^{\mu}(h(M \cdot))\right](\cdot) \frac{(-i \partial)^{\mu}}{\mu !} \hat{y}(0)=h(M \cdot) * \stackrel{y}{ }=0 \quad \forall h \in \Pi_{\kappa},
$$


and therefore, if and only if $\frac{(-i \partial)^{\mu}}{\mu !} \hat{y}(0)=0$ for all $|\mu| \leqslant \kappa$. In other words, $T_{u, M}(h *$ $y)=0$ for all $h \in \Pi_{\kappa}$ if and only if $\hat{y}\left(M^{T} \xi\right)=\hat{y}(\xi) \overline{\hat{u}(\xi)}^{T}=O\left(\|\xi\|^{\kappa+1}\right)$ as $\xi \rightarrow 0$. So, item (2) holds.

By (3.1), we have $T_{u, M} \mathcal{P}_{\kappa, y}=\mathcal{P}_{\kappa, \grave{y}}$ with $\hat{y}(\xi):=\hat{y}\left(\left(M^{T}\right)^{-1} \xi\right){\overline{\hat{u}\left(\left(M^{T}\right)^{-1} \xi\right)}}^{T}$. By Lemma 2.6. $\mathcal{P}_{\kappa, \grave{y}} \subseteq \mathcal{P}_{\kappa, y}$ if and only if (3.3) holds for some $2 \pi$-periodic trigonometric polynomial $\hat{c}$. Hence, item (3) is verified. It also follows from Lemma 2.6 that $\mathcal{P}_{\kappa, \grave{y}}=\mathcal{P}_{\kappa, y}$ if and only if (3.3) holds for some $2 \pi$-periodic trigonometric polynomial $\hat{c}$ with $\hat{c}(0) \neq 0$. Thus, item (4) holds.

Recall that $\Gamma_{M^{T}}$ is a complete set of representatives of the distinct cosets of $\left[\left(M^{T}\right)^{-1} \mathbb{Z}^{d}\right] / \mathbb{Z}^{d}$ with $0 \in \Gamma_{M^{T}}$. Denote by $\Omega_{M}$ a complete set of representatives of the distinct cosets of $\mathbb{Z}^{d} /\left[M \mathbb{Z}^{d}\right]$ with $0 \in \Omega_{M}$.

In order to study a subdivision operator $S_{u, M}$ acting on a space $\mathcal{P}_{\kappa, y}$, we need the following result, which generalizes [9, Proposition 2.2].

Lemma 3.2. Let $M$ be a $d \times d$ dilation matrix and $u: \mathbb{Z}^{d} \mapsto \mathbb{C}^{r \times r}$ be a finitely supported sequence of $r \times r$ matrices on $\mathbb{Z}^{d}$. Let $h \in \Pi^{1 \times r}$ be $a 1 \times r$ vector of polynomial sequences in $d$ variables. Then the following are equivalent:

(1) $S_{u, M} h$ is a $1 \times r$ vector of polynomial sequences; that is, $S_{u, M} h \in \Pi^{1 \times r}$.

(2) $\sum_{k \in \mathbb{Z}^{d}}\left(\partial^{\mu} h\right)\left(-M^{-1} \beta-k\right) u_{\beta+M k}=\sum_{k \in \mathbb{Z}^{d}}\left(\partial^{\mu} h\right)(-k) u_{M k}$ for all $\beta \in \Omega_{M}$ and $\mu \in \mathbb{N}_{0}^{d}$.

(3) $\left[\left(\partial^{\mu} h\right)(-i \partial) \widehat{u^{\beta}}\right](0)=\left[\left(\partial^{\mu} h\right)(-i \partial) \widehat{u^{0}}\right](0)$ for all $\beta \in \Omega_{M}$ and $\mu \in \mathbb{N}_{0}^{d}$, where $\widehat{u^{\beta}}(\xi):=\sum_{k \in \mathbb{Z}^{d}} u_{\beta+M k} e^{-i\left(M^{-1} \beta+k\right) \cdot \xi}$.

(4) $\left[\left(\partial^{\mu} h\right)\left(-i M^{-1} \partial\right) \hat{u}\right](2 \pi \gamma)=0$ for all $\gamma \in \Gamma_{M^{T}} \backslash\{0\}$ and $\mu \in \mathbb{N}_{0}^{d}$.

Moreover, if any of the above holds, then $S_{u, M} h=h\left(M^{-1} \cdot\right) * u$,

$$
S_{u, M}\left(\partial^{\nu} h\right)=\left[\left(\partial^{\nu} h\right)\left(M^{-1} \cdot\right)\right] * u \quad \forall \nu \in \mathbb{N}_{0}^{d},
$$

and

for all $k \in \mathbb{R}^{d}$.

$$
S_{u, M}(h(\cdot-k))=h\left(M^{-1} \cdot-k\right) * u
$$

Proof. By the definition of the subdivision operator $S_{u, M}$ in (1.17), it is easy to deduce that

$$
\left[S_{u, M} h\right]_{\beta+M j}=|\operatorname{det} M| \sum_{k \in \mathbb{Z}^{d}} h\left(M^{-1}(\beta+M j)-M^{-1} \beta-k\right) u_{\beta+M k}, \quad \beta, j \in \mathbb{Z}^{d} .
$$

Now we see that $S_{u, M} h \in \Pi^{1 \times r}$ if and only if $\sum_{k \in \mathbb{Z}^{d}} h\left(\cdot-M^{-1} \beta-k\right) u_{\beta+M k}$ is independent of $\beta$. Since

$$
h\left(x-M^{-1} \beta-k\right)=\sum_{\mu \in \mathbb{N}_{0}^{d}}\left(\partial^{\mu} h\right)\left(-M^{-1} \beta-k\right) \frac{x^{\mu}}{\mu !},
$$

we deduce that

$$
\sum_{k \in \mathbb{Z}^{d}} h\left(\cdot-M^{-1} \beta-k\right) u_{\beta+M k}=\sum_{\mu \in \mathbb{N}_{0}^{d}} \frac{x^{\mu}}{\mu !} \sum_{k \in \mathbb{Z}^{d}}\left(\partial^{\mu} h\right)\left(-M^{-1} \beta-k\right) u_{\beta+M k} .
$$

Thus, $\sum_{k \in \mathbb{Z}^{d}} h\left(\cdot-M^{-1} \beta-k\right) u_{\beta+M k}$ is independent of $\beta$ if and only if item (2) holds; that is, $\sum_{k \in \mathbb{Z}^{d}}\left(\partial^{\mu} h\right)\left(-M^{-1} \beta-k\right) u_{\beta+M k}$ is independent of $\beta$. So, (1) $\Leftrightarrow$ (2). 
To prove $(2) \Leftrightarrow(3)$, it suffices to show that

$$
\left[g(-i \partial) \widehat{u^{\beta}}\right](0)=\sum_{k \in \mathbb{Z}^{d}} g\left(-M^{-1} \beta-k\right) u_{\beta+M k}, \quad g \in \Pi^{1 \times r}, \beta \in \mathbb{Z}^{d} .
$$

Since $g(-i \partial)=\sum_{\mu \in \mathbb{N}_{0}^{d}} \partial^{\mu} g(0) \frac{(-i \partial)^{\mu}}{\mu !}$, we deduce that

$$
\begin{aligned}
{\left[g(-i \partial) \widehat{u^{\beta}}\right](0) } & =\sum_{\mu \in \mathbb{N}_{0}^{d}} \sum_{k \in \mathbb{Z}^{d}} \partial^{\mu} g(0) u_{\beta+M k}\left[\left.\frac{(-i \partial)^{\mu}}{\mu !} e^{\left.-i\left(M^{-1} \beta+k\right) \cdot \xi\right]}\right|_{\xi=0}\right. \\
& =\sum_{k \in \mathbb{Z}^{d}} \sum_{\mu \in \mathbb{N}_{0}^{d}} \partial^{\mu} g(0) \frac{\left(-M^{-1} \beta-k\right)^{\mu}}{\mu !} u_{\beta+M k} .
\end{aligned}
$$

By $g(x)=\sum_{\mu \in \mathbb{N}_{0}^{d}} \partial^{\mu} g(0) x^{\mu} / \mu$ !, we now see that (3.7) holds. So, (2) $\Leftrightarrow(3)$.

To prove $(3) \Leftrightarrow(4)$, we show that for $g \in \Pi^{1 \times r}$,

$$
\left[g(-i \partial) \widehat{u^{\beta}}\right](0)=g(-i \partial) \widehat{u^{0}}(0) \quad \forall \beta \in \Omega_{M}
$$

if and only if

$$
\left[g(-i \partial)\left(\hat{u}\left(\left(M^{T}\right)^{-1} \cdot+2 \pi \gamma\right)\right)\right](0)=0 \quad \forall \gamma \in \Gamma_{M^{T}} \backslash\{0\} .
$$

By $\hat{u}(\xi)=\sum_{\beta \in \Omega_{M}} \widehat{u^{\beta}}\left(M^{T} \xi\right)$, for $\gamma \in \Gamma_{M^{T}}$, we have

$$
\hat{u}\left(\left(M^{T}\right)^{-1} \xi+2 \pi \gamma\right)=\sum_{\beta \in \Omega_{M}} \widehat{u^{\beta}}(\xi) e^{-i 2 \pi \beta \cdot \gamma} .
$$

Putting these relations into a matrix form, we have

$$
\left.\left(\hat{u}\left(\left(M^{T}\right)^{-1} \xi+2 \pi \gamma\right)\right)_{\gamma \in \Gamma_{M^{T}}}=\left(e^{-i 2 \pi \beta \cdot \gamma}\right)_{\gamma \in \Gamma_{M^{T}}, \beta \in \Omega_{M}} \widehat{\left(u^{\beta}\right.}(\xi)\right)_{\beta \in \Omega_{M}} .
$$

Hence, we have

$\left(g(-i \partial)\left(\hat{u}\left(\left(M^{T}\right)^{-1} \cdot+2 \pi \gamma\right)\right)(0)\right)_{\gamma \in \Gamma_{M^{T}}}=\left(e^{-i 2 \pi \beta \cdot \gamma}\right)_{\gamma \in \Gamma_{M^{T}}, \beta \in \Omega_{M}}\left(g(-i \partial) \widehat{u^{\beta}}(0)\right)_{\beta \in \Omega_{M}}$.

Since $|\operatorname{det} M|^{-1 / 2}\left(e^{-i 2 \pi \beta \cdot \gamma}\right)_{\gamma \in \Gamma_{M^{T}}, \beta \in \Omega_{M}}$ is a unitary matrix, it is now easy to deduce that (3.8) is equivalent to (3.9). Since $g(-i \partial)\left(f\left(\left(M^{T}\right)^{-1} \cdot\right)\right)(0)=g\left(-i M^{-1} \partial\right) f(0)$, by (3.9), we conclude that $(3) \Leftrightarrow(4)$.

If any of (1)-(4) holds, by (3.6), we see that $S_{u, M} h=h\left(M^{-1} \cdot\right) * u$, since $h=\sum_{\beta \in \Omega_{M}} \sum_{k \in \mathbb{Z}^{d}} h\left(M^{-1}(\cdot-\beta-M k)\right) u_{\beta+M k}=\sum_{k \in \mathbb{Z}^{d}} h\left(M^{-1}(\cdot-k)\right) u_{k}=h\left(M^{-1} \cdot\right) * u$.

If item (2) holds for $h$, then it is straightforward to see that item (2) also holds with $h$ being replaced by $\partial^{\nu} h$. Consequently, (3.5) holds. Similarly, noting that $h(\cdot-k)=\sum_{\nu \in \mathbb{N}_{0}^{d}} \partial^{\nu} h(\cdot) \frac{(-k)^{\nu}}{\nu !}$, we see that $S_{u, M}(h(\cdot-k))=h\left(M^{-1} \cdot-k\right) * u$.

For a finitely supported sequence $u: \mathbb{Z}^{d} \mapsto \mathbb{C}$, by Lemma (3.2), we see that $S_{u, M} \Pi_{\kappa} \subseteq \Pi_{\kappa}$ if and only if

$$
\hat{u}(\xi+2 \pi \gamma)=O\left(\|\xi\|^{\kappa+1}\right), \quad \xi \rightarrow 0, \gamma \in \Gamma_{M^{T}} \backslash\{0\} .
$$

Similarly, by Lemma (3.2), we see that $S_{u, M} \Pi_{\kappa} \subseteq \Pi_{\kappa}$ if and only if $S_{u, M} p_{n} \in \Pi_{\kappa}$, $n=1, \ldots, N$ for some $p_{1}, \ldots, p_{N} \in \Pi_{\kappa}$ such that $\operatorname{span}\left\{\partial^{\mu} p_{n}: n=1, \ldots, N, \mu \in\right.$ $\left.\mathbb{N}_{0}^{d}\right\}=\Pi_{\kappa}$.

For a subdivision operator $S_{u, M}$ acting on a space $\mathcal{P}_{\kappa, y}$, we have the following result. 
Proposition 3.3. Let $u: \mathbb{Z}^{d} \mapsto \mathbb{C}^{r \times r}$ be a finitely supported sequence of $r \times r$ matrices on $\mathbb{Z}^{d}$. Let $y \in\left(\ell_{0}\left(\mathbb{Z}^{d}\right)\right)^{1 \times r}$ be a finitely supported sequence of $1 \times r$ vectors on $\mathbb{Z}^{d}$. Then for any nonnegative integer $\kappa$, the following statements hold:

(1) $S_{u, M} \mathcal{P}_{\kappa, y} \subseteq\left(\Pi_{\kappa}\right)^{1 \times r}$ if and only if

$$
\hat{y}\left(M^{T} \xi\right) \hat{u}(\xi+2 \pi \gamma)=O\left(\|\xi\|^{\kappa+1}\right), \quad \xi \rightarrow 0, \gamma \in \Gamma_{M^{T}} \backslash\{0\} .
$$

(2) If (3.12) holds, define $\hat{\dot{y}}(\xi):=\hat{y}\left(M^{T} \xi\right) \hat{u}(\xi)$. Then $S_{u, M} \mathcal{P}_{\kappa, y}=\mathcal{P}_{\kappa, \grave{y}}$ and

$$
\begin{aligned}
S_{u, M}(h * y) & =|\operatorname{det} M|^{-1} S_{S_{u, M} y, M} h=|\operatorname{det} M|^{-1} h\left(M^{-1} \cdot\right) *\left[S_{u, M} y\right] \\
& =h\left(M^{-1} \cdot\right) * \dot{y}, \quad h \in \Pi_{\kappa} .
\end{aligned}
$$

If in addition $\hat{y}(0) \hat{u}(0) \neq 0$, then mapping $\left.S_{u, M}\right|_{\mathcal{P}_{\kappa, y}}: \mathcal{P}_{\kappa, y} \mapsto \mathcal{P}_{\kappa, \hat{y}}$ is oneto-one and onto.

(3) $S_{u, M} T_{v, M}(h * y)=h * y$ for all $h \in \Pi_{\kappa}$ if and only if

$$
\hat{y}(\xi) \overline{\hat{v}(\xi)}^{T} \hat{u}(\xi+2 \pi \gamma)=\delta_{\gamma} \hat{y}(\xi)+O\left(\|\xi\|^{\kappa+1}\right), \quad \xi \rightarrow 0, \gamma \in \Gamma_{M^{T}} .
$$

Proof. Denote $\hat{y}(\xi):=\hat{y}\left(M^{T} \xi\right) \hat{u}(\xi)$. To prove items (1) and (2), we first show that we always have

$$
S_{u, M}(h * y)=|\operatorname{det} M|^{-1} S_{S_{u, M} y, M} h=S_{\grave{y}, M} h, \quad h \in \Pi .
$$

In fact, by the definition of the subdivision operator in (1.17), we deduce that

$$
\begin{aligned}
{\left[S_{u, M}(h * y)\right]_{j} } & =|\operatorname{det} M| \sum_{k \in \mathbb{Z}^{d}}(h * y)_{k} u_{j-M k} \\
& =|\operatorname{det} M| \sum_{k \in \mathbb{Z}^{d}} \sum_{n \in \mathbb{Z}^{d}} h(k-n) y_{n} u_{j-M k} \\
& =|\operatorname{det} M| \sum_{k \in \mathbb{Z}^{d}} \sum_{n \in \mathbb{Z}^{d}} h(k) y_{n} u_{j-M(k-n)} \\
& =\sum_{k \in \mathbb{Z}^{d}} h(k)|\operatorname{det} M| \sum_{n \in \mathbb{Z}^{d}} y_{n} u_{j-M k-M n} \\
& =\sum_{k \in \mathbb{Z}^{d}} h(k)\left[S_{u, M} y\right]_{j-M k}=|\operatorname{det} M|^{-1}\left[S_{S_{u, M} y, M} h\right]_{j} .
\end{aligned}
$$

Since $\widehat{S_{u, M} y}(\xi)=|\operatorname{det} M| \hat{y}\left(M^{T} \xi\right) \hat{u}(\xi)=|\operatorname{det} M| \hat{y}(\xi)$, we now see that (3.14) is verified. In particular, by (3.14), $S_{u, M} \mathcal{P}_{\kappa, y}=S_{\grave{y}, M} \Pi_{\kappa}$. By Lemma 3.2 and in particular (3.11), we conclude that $S_{u, M} \mathcal{P}_{\kappa, y}=S_{\grave{y}, M} \Pi_{\kappa} \subseteq\left(\Pi_{\kappa}\right)^{1 \times r}$ if and only if $\hat{y}(\xi+2 \pi \gamma)=O\left(\|\xi\|^{\kappa+1}\right)$ as $\xi \rightarrow 0$ for all $\gamma \in \Gamma_{M^{T}} \backslash\{0\}$. That is, $S_{u, M} \mathcal{P}_{\kappa, y} \subseteq\left(\Pi_{\kappa}\right)^{1 \times r}$ if and only if (3.12) holds. So, item (1) is verified. Item (2) is a direct consequence of (3.14) and Lemma 3.2 If $\hat{\hat{y}}(0)=\hat{y}(0) \hat{u}(0) \neq 0$, by Proposition 2.1, then we have $\operatorname{dim}\left(\mathcal{P}_{\kappa, y}\right)=\operatorname{dim}\left(\mathcal{P}_{\kappa, \grave{y}}\right)=\operatorname{dim}\left(\Pi_{\kappa}\right)$. Now it follows from $S_{u, M} \mathcal{P}_{\kappa, y}=\mathcal{P}_{\kappa, \grave{y}}$ that the restricted mapping $\left.S_{u, M}\right|_{\mathcal{P}_{\kappa, y}}: \mathcal{P}_{\kappa, y} \mapsto \mathcal{P}_{\kappa, \dot{y}}$ is one-to-one and onto.

To prove item (3), by (3.1), we have $T_{v, M}(h * y)=h(M \cdot) * \tilde{y}, h \in \Pi_{\kappa}$ with $\hat{\tilde{y}}\left(M^{T} \xi\right):=\hat{y}(\xi) \overline{\hat{v}}(\xi)^{T}+O\left(\|\xi\|^{\kappa+1}\right), \xi \rightarrow 0$. Therefore, $S_{u, M} T_{v, M}(h * y)=h * y$ for all $h \in \Pi_{\kappa}$ if and only if

$$
S_{u, M}(h(M \cdot) * \tilde{y})=h * y, \quad h \in \Pi_{\kappa} .
$$

Now by items (1) and (2), (3.15) holds if and only if

$$
\hat{\tilde{y}}\left(M^{T} \xi\right) \hat{u}(\xi+2 \pi \gamma)=\hat{y}(\xi) \overline{\hat{v}(\xi)}^{T} \hat{u}(\xi+2 \pi \gamma)=O\left(\|\xi\|^{\kappa+1}\right), \quad \xi \rightarrow 0, \gamma \in \Gamma_{M^{T}} \backslash\{0\}
$$


and

$$
\hat{\tilde{y}}\left(M^{T} \xi\right) \hat{u}(\xi)=\hat{y}(\xi) \overline{\hat{v}(\xi)}^{T} \hat{u}(\xi)=\hat{y}(\xi)+O\left(\|\xi\|^{\kappa+1}\right), \quad \xi \rightarrow 0 .
$$

Therefore, (3.15) holds if and only if (3.13) holds. Hence, item (3) is verified.

Lemma 3.4. Let $u \in\left(\ell_{0}\left(\mathbb{Z}^{d}\right)\right)^{r \times r}$ and $y, \tilde{y} \in\left(\ell_{0}\left(\mathbb{Z}^{d}\right)\right)^{1 \times r}$ with $\hat{\tilde{y}}(0) \neq 0$. Then $S_{u, M} \mathcal{P}_{\kappa, y}=\mathcal{P}_{\kappa, \tilde{y}}$ if and only if

$$
\hat{y}\left(M^{T} \xi\right) \hat{u}(\xi+2 \pi \gamma)=\delta_{\gamma} \hat{c}(\xi) \hat{\tilde{y}}(\xi)+O\left(\|\xi\|^{\kappa+1}\right), \quad \xi \rightarrow 0, \gamma \in \Gamma_{M^{T}}
$$

for some $2 \pi$-periodic trigonometric polynomial $\hat{c}$ with $\hat{c}(0) \neq 0$. In particular, for $y \in\left(\ell_{0}\left(\mathbb{Z}^{d}\right)\right)^{1 \times r}$ with $\hat{y}(0) \neq 0, S_{u, M} \mathcal{P}_{\kappa, y}=\mathcal{P}_{\kappa, y}$ if and only if

$$
\begin{gathered}
{\left[\hat{\tilde{c}}\left(M^{T} \xi\right) \hat{y}\left(M^{T} \xi\right)\right][C \hat{u}(\xi+2 \pi \gamma)]=\delta_{\gamma}[\hat{\tilde{c}}(\xi) \hat{y}(\xi)]+O\left(\|\xi\|^{\kappa+1}\right),} \\
\xi \rightarrow 0, \gamma \in \Gamma_{M^{T}}
\end{gathered}
$$

for some nonzero constant $C$ and some $2 \pi$-periodic trigonometric polynomial $\hat{\tilde{c}}$ with $\hat{\tilde{c}}(0) \neq 0$.

Proof. If (3.16) holds, then it is evident that (3.12) holds and

$$
\hat{\hat{y}}(\xi):=\hat{y}\left(M^{T} \xi\right) \hat{u}(\xi)=\hat{c}(\xi) \hat{\tilde{y}}(\xi)+O\left(\|\xi\|^{\kappa+1}\right), \quad \xi \rightarrow 0 .
$$

So, by item (2) of Proposition 3.3 and Lemma 2.6, we conclude that $S_{u, M} \mathcal{P}_{\kappa, y}=$ $\mathcal{P}_{\kappa, \check{y}}=\mathcal{P}_{\kappa, \tilde{y}}$.

Conversely, if $S_{u, M} \mathcal{P}_{\kappa, y}=\mathcal{P}_{\kappa, \tilde{y}}$, then by items (1) and (2) of Proposition 3.3. we conclude that (3.12) holds and $S_{u, M} \mathcal{P}_{\kappa, y}=\mathcal{P}_{\kappa, \grave{y}}$. Since $S_{u, M} \mathcal{P}_{\kappa, y}=\mathcal{P}_{\kappa, \tilde{y}}$, this implies that $\mathcal{P}_{\kappa, \tilde{y}}=\mathcal{P}_{\kappa, \tilde{y}}$. Since $\hat{\tilde{y}}(0) \neq 0$, by Lemma 2.6, there must exist a $2 \pi$ periodic trigonometric polynomial $\hat{c}$ with $\hat{c}(0) \neq 0$ such that (3.18) holds. Now it is straightforward to see that (3.12) and (3.18) are equivalent to (3.16).

We now prove the second claim. By what has been proved, $S_{u, M} \mathcal{P}_{\kappa, y}=\mathcal{P}_{\kappa, y}$ if and only if

$$
\hat{y}\left(M^{T} \xi\right) \hat{u}(\xi+2 \pi \gamma)=\delta_{\gamma} \hat{c}(\xi) \hat{y}(\xi)+O\left(\|\xi\|^{\kappa+1}\right), \quad \xi \rightarrow 0, \gamma \in \Gamma_{M^{T}}
$$

for some $2 \pi$-periodic trigonometric polynomial $\hat{c}$ with $\hat{c}(0) \neq 0$. Since $\hat{c}(0) \neq 0$, by Lemma 2.5, (2.17) holds. Take $\hat{\tilde{c}}=\widehat{c^{2}}$ and $C=1 / \hat{c}(0)$. We see that (3.19) holds if and only if (3.17) holds.

We mention that (3.17) (or (3.19)) is equivalent to saying that the mask $C \hat{u}(\xi)$ (or the mask $\hat{u}(\xi) / \hat{c}(0))$ satisfies the sum rules of order $\kappa+1$ in (2.2) with $\hat{a}$ and $\hat{y}$ being replaced by $C \hat{u}$ and $\hat{\tilde{c}} \hat{y}$ (or by $\hat{u} / \hat{c}(0)$ and $\widehat{c^{2}} \hat{y}$ with $\widehat{c^{2}}$ in (2.17)), respectively. More precisely, we have the following result:

Corollary 3.5. Let $u \in\left(\ell_{0}\left(\mathbb{Z}^{d}\right)\right)^{r \times r}$ and $y \in\left(\ell_{0}\left(\mathbb{Z}^{d}\right)\right)^{1 \times r}$ with $\hat{y}(0) \neq 0$. Then the following are equivalent:

(1) $\hat{u}$ has $\kappa+1$ sum rules with the dilation matrix $M$ and the vector sequence $y$ in (3.17) with $C=1$ for some $2 \pi$-periodic trigonometric polynomial $\hat{\tilde{c}}$ with $\hat{\tilde{c}}(0) \neq 0$.

(2) $\hat{u}$ satisfies (3.19) for some $2 \pi$-periodic trigonometric polynomial $\hat{c}$ with $\hat{c}(0)=1$.

(3) $\hat{y}(0) \hat{u}(0)=\hat{y}(0)$ and $S_{u, M} \mathcal{P}_{\kappa, y}=\mathcal{P}_{\kappa, y}$.

(4) $\hat{y}(0) \hat{u}(0)=\hat{y}(0)$ and there are polynomials $h_{n} \in \Pi_{\kappa}, n=1, \ldots, N$, such that $S_{u, M}\left(h_{n} * y\right) \in \mathcal{P}_{\kappa, y}$ for all $n=1, \ldots, N$ and $\operatorname{span}\left\{\partial^{\mu} h_{n}: \mu \in \mathbb{N}_{0}^{d}, n=\right.$ $1, \ldots, N\}=\Pi_{\kappa}$. 


\section{BALANCING AND PREFILTERING PROPERTY OF DUAL MULTIFRAMELETS}

Based on the results in previous sections, we shall discuss in this section the prefiltering and balancing property of multivariate dual multiframelets and biorthogonal multiwavelets from the point of view of discrete algorithms using transition operators and subdivision operators.

For dual multiframelets, we have the following main result in this section.

Theorem 4.1. Let $M$ denote a $d \times d$ dilation matrix and $\kappa \in \mathbb{N}_{0}$. Let $\hat{a}, \widehat{b^{1}}, \ldots, \widehat{b^{L}}$ and $\hat{\tilde{a}}, \widehat{\tilde{b}^{1}}, \ldots, \widehat{\tilde{b}^{L}}$ be $r \times r$ matrices of $2 \pi$-periodic trigonometric polynomials in $d$ variables such that (1.9) is satisfied. Let $E: \ell\left(\mathbb{Z}^{d}\right) \mapsto\left(\ell\left(\mathbb{Z}^{d}\right)\right)^{1 \times r}$ be a vector conversion operator. Let $y \in\left(\ell_{0}\left(\mathbb{Z}^{d}\right)\right)^{1 \times r}$ be given by Proposition 2.2 such that $\mathcal{P}_{\kappa, y}=E\left(\Pi_{\kappa}\right)$. If the associated multiframelet transform has $\kappa+1$ balancing order with respect to the vector conversion operator $E$, then

1) there is a $2 \pi$-periodic trigonometric polynomial $\hat{c}$ such that

$$
\hat{y}(\xi) \overline{\tilde{\tilde{a}}}(\xi)^{T}=\hat{c}(\xi) \hat{y}\left(M^{T} \xi\right)+O\left(\|\xi\|^{\kappa+1}\right), \quad \xi \rightarrow 0
$$

2) The high-pass filters $\widehat{\tilde{b}^{1}}, \ldots, \widehat{\tilde{b}}^{L}$ have $\kappa+1$ discrete vanishing moments with respect to $\hat{y}$ :

$$
\hat{y}(\xi){\overline{\tilde{b}^{\ell}(\xi)}}^{T}=O\left(\|\xi\|^{\kappa+1}\right), \quad \xi \rightarrow 0, \ell=1, \ldots, L .
$$

3) $S_{a, M} T_{\tilde{a}, M}(h * y)=h * y$ for all $h \in \Pi_{\kappa}$.

4) $T_{\tilde{a}, M} \mathcal{P}_{\kappa, y}=\mathcal{P}_{\kappa, y}$. That is, (4.1) holds with $\hat{c}(0) \neq 0$.

5) $S_{a, M} \mathcal{P}_{\kappa, y}=\mathcal{P}_{\kappa, y}$.

Conversely, items 1) and 2) together imply that the multiframelet transform has $\kappa+1$ balancing order with respect to the vector conversion operator $E$. Moreover, if $L=|\operatorname{det} M|-1$ (this is true for a biorthogonal multiwavelet), then the associated multiframelet transform has $\kappa+1$ balancing order with respect to $E$ if and only if item 5) holds.

Proof. First, we show that the multiframelet transform has $\kappa+1$ balancing order with respect to $E$ if and only if items 1) and 2) hold. Since $E\left(\Pi_{\kappa}\right)=\mathcal{P}_{\kappa, y}$, by item (2) of Proposition 3.1 $T_{\tilde{b}^{\ell}, M} E\left(\Pi_{\kappa}\right)=T_{\tilde{b}^{\ell}, M} \mathcal{P}_{\kappa, y}=0$ for all $\ell=1, \ldots, L$ if and only if item 2) holds. Similarly, by item (1) of Proposition 3.1, we see that $T_{\tilde{a}, M} E\left(\Pi_{\kappa}\right)=T_{\tilde{a}, M} \mathcal{P}_{\kappa, y}=\mathcal{P}_{\kappa, \tilde{y}}$ with

$$
\hat{\grave{y}}\left(M^{T} \xi\right):=\hat{y}(\xi) \overline{\tilde{\tilde{a}}}(\xi)^{T} .
$$

Hence, $T_{\tilde{a}, M} \mathcal{P}_{\kappa, y} \subseteq \mathcal{P}_{\kappa, y}$ if and only if $\mathcal{P}_{\kappa, y} \subseteq \mathcal{P}_{\kappa, y}$. Since $\hat{y}(0) \neq 0$, by Lemma 2.6. we conclude that $T_{\tilde{a}, M} \mathcal{P}_{\kappa, y} \subseteq \mathcal{P}_{\kappa, y}$ if and only if (4.1) holds. Therefore, the multiframelet transform has $\kappa+1$ balancing order with respect to $E$ if and only if items 1) and 2) hold.

Suppose that the multiframelet transform has $\kappa+1$ balancing order. Then items 1) and 2) hold. We now prove items 3), 4) and 5). From the relation in (1.9), we deduce that

$$
[\hat{y}(\xi), 0, \ldots, 0]{\overline{P_{\left[\hat{a}, \widehat{\vec{b}}^{1}, \ldots, \widehat{\tilde{b}^{L}}\right]}(\xi)}}^{T} P_{\left[\hat{a}, \widehat{b}^{1}, \ldots, \widehat{b^{L}}\right]}(\xi)=[\hat{y}(\xi), 0 \ldots, 0] .
$$


By calculation, the above relation is equivalent to

$$
\hat{y}(\xi) \overline{\tilde{\tilde{a}}}^{T} \hat{x} \hat{a}(\xi+2 \pi \gamma)+\sum_{\ell=1}^{L} \hat{y}(\xi) \overline{\hat{\tilde{b}}}^{\ell}(\xi) \widehat{b}^{\ell}(\xi+2 \pi \gamma)=\delta_{\gamma} \hat{y}(\xi), \quad \gamma \in \Gamma_{M^{T}} .
$$

Now by item 2), it follows from the above relation that

$$
\hat{y}(\xi) \overline{\hat{\tilde{a}}}(\xi)^{T} \hat{a}(\xi+2 \pi \gamma)=\delta_{\gamma} \hat{y}(\xi)+O\left(\|\xi\|^{\kappa+1}\right), \quad \xi \rightarrow 0, \gamma \in \Gamma_{M^{T}} .
$$

Thus, by item (3) of Proposition 3.3. we conclude that item 3) holds.

In particular, item 3) implies that $S_{a, M} T_{\tilde{a}, M} \mathcal{P}_{k, y}=\mathcal{P}_{\kappa, y}$. Since both $S_{a, M}$ and $T_{\tilde{a}, M}$ are linear operators, $S_{a, M} T_{\tilde{a}, M} \mathcal{P}_{k, y}=\mathcal{P}_{\kappa, y}$ and $T_{\tilde{a}, M} \mathcal{P}_{\kappa, y} \subseteq \mathcal{P}_{\kappa, y}$ (this is item (i) in the definition of $\kappa+1$ balancing order) will force $\operatorname{dim}\left(T_{\tilde{a}, M} \mathcal{P}_{\kappa, y}\right)=\operatorname{dim}\left(\mathcal{P}_{\kappa, y}\right)$. That is, we must have $T_{\tilde{a}, M} \mathcal{P}_{\kappa, y}=\mathcal{P}_{\kappa, y}$. Now we have $\mathcal{P}_{\kappa, \tilde{y}}=T_{\tilde{a}, M} \mathcal{P}_{\kappa, y}=\mathcal{P}_{\kappa, y}$, where $\hat{\dot{y}}$ is defined in (4.3). By Lemma 2.6. (4.1) must hold with $\hat{c}(0) \neq 0$. Hence, item 4) holds.

By items 3) and 4), it is straightforward to see that $S_{a, M} \mathcal{P}_{\kappa, y}=\mathcal{P}_{\kappa, y}$. Thus, item 5) holds.

For the case $L=|\operatorname{det} M|-1$, in order to show that the multiframelet transform has $\kappa+1$ balancing order if and only if item 5 ) holds, we only need to prove that item 5) implies both items 1) and 2). By $\hat{y}(0) \neq 0$ and Lemma 3.4, we first note that item 5) is equivalent to

$$
\left[\hat{\hat{c}}\left(M^{T} \xi\right) \hat{y}\left(M^{T} \xi\right)\right][C \hat{a}(\xi+2 \pi \gamma)]=\delta_{\gamma}[\hat{c}(\xi) \hat{y}(\xi)]+O\left(\|\xi\|^{\kappa+1}\right), \quad \xi \rightarrow 0, \gamma \in \Gamma_{M^{T}}
$$

for some nonzero number $C \in \mathbb{C} \backslash\{0\}$ and some $2 \pi$-periodic trigonometric polynomial $\hat{c}$ with $\hat{c}(0) \neq 0$. When $L=|\operatorname{det} M|-1$, the matrices $P_{\left[\hat{a}, \widehat{b^{1}}, \ldots, \widehat{b^{L}}\right]}$ and $P_{\left[\hat{\tilde{a}}, \widehat{b^{1}}, \ldots, \widehat{b^{L}}\right]}$ are square matrices. Therefore, (1.9) implies that

$$
P_{\left[\hat{a}, \widehat{b^{1}}, \ldots, \widehat{b^{L}}\right]}(\xi){\overline{P_{\left[\hat{\tilde{a}}, \widehat{\widehat{b}^{1}}, \ldots, \widehat{\tilde{b}^{L}}\right]}(\xi)}}^{T}=I_{m r}
$$

Hence, we have

$$
\begin{aligned}
\left(C \hat{\hat{c}}\left(M^{T} \xi\right) \hat{y}\left(M^{T} \xi\right), 0, \ldots, 0\right) P_{\left[\hat{a}, \widehat{b^{1}}, \ldots, \widehat{b^{L}}\right]}(\xi){\overline{P_{\left[\hat{a}, \widehat{b^{1}}, \ldots, \widehat{b^{L}}\right]}(\xi)}}^{T} \\
=\left(C \hat{\grave{c}}\left(M^{T} \xi\right) \hat{y}\left(M^{T} \xi\right), 0, \ldots, 0\right) .
\end{aligned}
$$

By calculation, the above identity is equivalent to

$$
\begin{aligned}
& \sum_{\gamma \in \Gamma_{M^{T}}} C \hat{\hat{c}}\left(M^{T} \xi\right) \hat{y}\left(M^{T} \xi\right) \hat{a}(\xi+2 \pi \gamma) \overline{\overline{\tilde{a}}(\xi+2 \pi \gamma)}{ }^{T}=C \hat{\grave{c}}\left(M^{T} \xi\right) \hat{y}\left(M^{T} \xi\right), \\
& \sum_{\gamma \in \Gamma_{M^{T}}} C \hat{\hat{c}}\left(M^{T} \xi\right) \hat{y}\left(M^{T} \xi\right) \hat{a}(\xi+2 \pi \gamma){\overline{\hat{\tilde{b}}^{\ell}(\xi+2 \pi \gamma)}}^{T}=0, \quad \ell=1, \ldots, L .
\end{aligned}
$$

By (4.5) and the above two identities, we deduce that

$$
\begin{aligned}
& \hat{\hat{c}}(\xi) \hat{y}(\xi) \overline{\hat{\tilde{a}}(\xi)}^{T}=C \hat{\hat{c}}\left(M^{T} \xi\right) \hat{y}\left(M^{T} \xi\right)+O\left(\|\xi\|^{\kappa+1}\right) \quad \text { and } \\
& \hat{\dot{c}}(\xi) \hat{y}(\xi) \overline{\hat{\tilde{b}}}^{\ell}(\xi)=O\left(\|\xi\|^{\kappa+1}\right), \quad \xi \rightarrow 0 .
\end{aligned}
$$

Since $\hat{\grave{c}}(0) \neq 0$, we now see that (4.1) and (4.2) hold with $\hat{c}(\xi)=C \hat{\grave{c}}\left(M^{T} \xi\right) / \hat{\grave{c}}(\xi)$. 
In fact, the two $2 \pi$-periodic trigonometric polynomials $\hat{c}$ in (4.1) and $\hat{c}$ in (4.5) are related and determined by each other through the following relation:

$$
\hat{c}(\xi) \hat{c}(\xi)=C \hat{\dot{c}}\left(M^{T} \xi\right)+O\left(\|\xi\|^{\kappa+1}\right), \quad \xi \rightarrow 0 \quad \text { and } \quad C=\hat{c}(0) .
$$

In fact, with $\gamma=0$ in (4.5), we have

$$
\hat{y}\left(M^{T} \xi\right) \hat{a}(\xi)=C^{-1} \frac{\hat{c}(\xi)}{\hat{c}\left(M^{T} \xi\right)} \hat{y}(\xi)+O\left(\|\xi\|^{\kappa+1}\right), \quad \xi \rightarrow 0 .
$$

Now by (4.1) and the above relation, it follows from (4.4) with $\gamma=0$ that

$$
\begin{aligned}
\hat{y}(\xi) & =\hat{y}(\xi) \overline{\hat{\tilde{a}}}(\xi)^{T} \hat{a}(\xi)+O\left(\|\xi\|^{\kappa+1}\right)=\hat{c}(\xi) \hat{y}\left(M^{T} \xi\right) \hat{a}(\xi)+O\left(\|\xi\|^{\kappa+1}\right) \\
& =C^{-1} \hat{c}(\xi) \frac{\hat{\grave{c}}(\xi)}{\hat{\hat{c}}\left(M^{T} \xi\right)} \hat{y}(\xi)+O\left(\|\xi\|^{\kappa+1}\right), \quad \xi \rightarrow 0 .
\end{aligned}
$$

Since $\hat{y}(0) \neq 0$, it is now easy to see that

$$
C^{-1} \hat{c}(\xi) \frac{\hat{c}(\xi)}{\hat{c}\left(M^{T} \xi\right)}=1+O\left(\|\xi\|^{\kappa+1}\right)
$$

as $\xi \rightarrow 0$. So, (4.6) holds. Note that (1.9) still holds if $\hat{a}$ and $\hat{\tilde{a}}$ are replaced with $C \hat{a}$ and $\overline{C^{-1}} \hat{\tilde{a}}$, respectively. Therefore, the appearance of a nonzero constant $C$ in (4.5) is very natural, due to the lack of normalization for $\hat{a}$ and $\hat{\tilde{a}}$ in (1.9). Without loss of any generality, one may assume that $C=1$ and consequently, by (4.1) and (4.5), $\hat{y}(0) \overline{\hat{\tilde{a}}(0)}^{T}=\hat{y}(0)=\hat{y}(0) \hat{a}(0)$; that is, $\hat{y}(0)$ is a nonzero left eigenvector to both $\hat{a}(0)$ and $\overline{\hat{\tilde{a}}}(0)^{T}$ with the eigenvalue 1 .

From the following known result (e.g., [9, Proposition 3.1]), which is a direct consequence of [9, Lemma 2.2], we shall see that under some mild conditions the space $\mathcal{P}_{\kappa, y}$ is generally uniquely determined by the values $\partial^{\mu} \hat{a}(0),|\mu| \leqslant \kappa$ or by the values $\partial^{\mu} \hat{\tilde{a}}(0),|\mu| \leqslant \kappa$ if $\hat{\tilde{a}}$ is a dual mask of $\hat{a}$.

Proposition 4.2. Let $M$ be a $d \times d$ dilation matrix and denote $\sigma:=\left(\sigma_{1}, \ldots, \sigma_{d}\right)^{T}$, where $\sigma_{1}, \ldots, \sigma_{d}$ are all the eigenvalues of $M$. Let $\hat{a}$ and $\hat{\tilde{a}}$ be $r \times r$ matrices of $2 \pi$-periodic trigonometric polynomials in $d$ variables.

(1) If 1 is a simple eigenvalue of $\hat{a}(0)$ and $\sigma^{-\mu}, 0<|\mu| \leqslant \kappa$ and $\mu \in \mathbb{N}_{0}^{d}$, are not eigenvalues of $\hat{a}(0)$, then up to a multiplicative constant, there is a unique nontrivial solution $\left\{\partial^{\mu} \hat{y}(0):|\mu| \leqslant \kappa\right\}$ to the system of linear equations induced by

$$
\hat{y}\left(M^{T} \xi\right) \hat{a}(\xi)=\hat{y}(\xi)+O\left(\|\xi\|^{\kappa+1}\right), \quad \xi \rightarrow 0 .
$$

(2) If 1 is a simple eigenvalue of $\hat{\tilde{a}}(0)$ and $\sigma^{\mu}, 0<|\mu| \leqslant \kappa$ and $\mu \in \mathbb{N}_{0}^{d}$, are not eigenvalues of $\hat{\tilde{a}}(0)$, then up to a multiplicative constant, there is a unique nontrivial solution $\left\{\partial^{\mu} \hat{\tilde{\phi}}(0):|\mu| \leqslant \kappa\right\}$ to the system of linear equations induced by

$$
\hat{\tilde{\phi}}\left(M^{T} \xi\right)=\hat{\tilde{a}}(\xi) \hat{\tilde{\phi}}(\xi)+O\left(\|\xi\|^{\kappa+1}\right), \quad \xi \rightarrow 0 .
$$

In particular, suppose that $\hat{a}$ has $\kappa+1$ sum rules in (2.2) with a vector $y \in$ $\left(\ell_{0}\left(\mathbb{Z}^{d}\right)\right)^{1 \times r}$. Then 
(i) if the condition in item (1) holds for $\hat{a}(0)$, then up to a multiplicative constant the values $\partial^{\mu} \hat{y}(0),|\mu| \leqslant \kappa$ and the space $\mathcal{P}_{\kappa, y}$ are uniquely determined by the values $\partial^{\mu} \hat{a}(0),|\mu| \leqslant \kappa$;

(ii) if the condition in item (2) holds for $\hat{\tilde{a}}(0)$ and if $\hat{\tilde{a}}$ is a dual mask of $\hat{a}$, that is,

$$
\sum_{\gamma \in \Gamma_{M^{T}}} \hat{a}(\xi+2 \pi \gamma) \overline{\hat{\tilde{a}}(\xi+2 \pi \gamma)}^{T}=1,
$$

then up to a multiplicative constant the values $\partial^{\mu} \hat{y}(0),|\mu| \leqslant \kappa$ and the space $\mathcal{P}_{\kappa, y}$ are uniquely determined by the values $\partial^{\mu} \hat{\tilde{a}}(0),|\mu| \leqslant \kappa$ via the following formula:

$$
\hat{y}(\xi) \overline{\hat{\tilde{a}}(\xi)}^{T}=\hat{y}\left(M^{T} \xi\right)+O\left(\|\xi\|^{\kappa+1}\right), \quad \xi \rightarrow 0 .
$$

Proof. Item (ii) has been proved in [8. Theorem 3.1] and plays an important role in the CBC (coset by coset) algorithm for constructing biorthogonal multiwavelets with arbitrarily high orders of vanishing moments. For completeness, we sketch the proof here in the frequency domain. By (4.7) and (2.2), we can easily deduce that $\hat{y}\left(M^{T} \xi\right)=\sum_{\gamma \in \Gamma_{M^{T}}} \hat{y}\left(M^{T} \xi\right) \hat{a}(\xi+2 \pi \gamma) \overline{\tilde{\tilde{a}}}(\xi+2 \pi \gamma)^{T}=\hat{y}(\xi) \overline{\tilde{\tilde{a}}}(\xi)^{T}+O\left(\|\xi\|^{\kappa+1}\right), \quad \xi \rightarrow 0$.

Hence, (4.8) holds. By the condition in item (2) for $\hat{\tilde{a}}(0)$ and [9. Lemma 2.2], up to a multiplicative constant, there is a unique nontrivial solution $\left\{\partial^{\mu} \hat{y}(0):|\mu| \leqslant \kappa\right\}$ to the system of linear equations induced by (4.8).

By Theorem 4.1 and Proposition 4.2, we see that the space $\mathcal{P}_{\kappa, y}$ is generally determined by either a vector conversion operator $E$ or a mask $\hat{a}$. Namely, we have two choices to determine the space $\mathcal{P}_{\kappa, y}$ : one corresponds to balanced multiframelets by fixing $E$ and designing $\hat{a}$, and the other corresponds to prefiltering a given dual multiframelet by fixing $\hat{a}$ and designing $E$.

One choice is to fix a vector conversion operator $E$, for example, taking $E=E_{r}$, the standard vector conversion operator in (1.22). By Proposition 2.2, we have $E\left(\Pi_{\kappa}\right)=\mathcal{P}_{\kappa, y}$ for some $y \in\left(\ell_{0}\left(\mathbb{Z}^{d}\right)\right)^{1 \times r}$ with $\hat{y}(0) \neq 0$. Note that if $E=E_{r}$, then $\hat{y}$ can be taken to be $\hat{Y}$ in (2.9). Then by Theorem 4.1, the design of balanced multiwavelets and multiframelets with $\kappa+1$ balancing order with respect to the given vector conversion operator $E$ (often $E=E_{r}$ ) corresponds to designing

(1) a mask $\hat{a}$ such that $\hat{a}$ has $\kappa+1$ sum rules in (4.5) with $C=1$, where $\hat{c}$ can be freely chosen to be any $2 \pi$-periodic trigonometric polynomial with $\hat{\grave{c}}(0) \neq 0$;

(2) a mask $\hat{\tilde{a}}$ such that (4.1) holds with $\hat{c}(\xi):=\hat{\grave{c}}\left(M^{T} \xi\right) / \hat{\grave{c}}(\xi)$;

(3) high-pass filters $\widehat{b^{1}}, \ldots, \widehat{b^{L}}, \widehat{\tilde{b}^{1}}, \ldots, \widehat{\tilde{b}^{L}}$ such that (1.9) and (4.2) hold.

The above three conditions can be translated into the function setting as follows:

Proposition 4.3. Assume that there are compactly supported $1 \times r$ function vectors $\phi, \psi^{1}, \ldots, \psi^{L}, \tilde{\phi}, \tilde{\psi}^{1}, \ldots, \tilde{\psi}^{L}$ in $L_{2}\left(\mathbb{R}^{d}\right)$ such that (1.11) and (1.12) are satisfied. Suppose that (4.5) holds with $\gamma=0$ and $C=1$. We further assume that the condition on $\hat{\tilde{a}}$ in item (2) of Proposition 4.2 is satisfied. Then the associated multiframelet transform has $\kappa+1$ balancing order with respect to a vector conversion operator $E$ satisfying $E\left(\Pi_{\kappa}\right)=\mathcal{P}_{\kappa, y}$ if and only if 
(1) for some $2 \pi$-periodic trigonometric polynomial $\hat{\tilde{c}}$ with $\hat{\tilde{c}}(0) \neq 0$,

$$
\hat{\tilde{c}}(\xi) \hat{y}(\xi)=\overline{\hat{\tilde{\phi}}}^{T}(\xi)+O\left(\|\xi\|^{\kappa+1}\right), \quad \xi \rightarrow 0 ;
$$

(2) all wavelet function vectors $\tilde{\psi}^{1}, \ldots, \tilde{\psi}^{L}$ have $\kappa+1$ vanishing moments:

$$
\widehat{\tilde{\psi}^{\ell}}(\xi)=O\left(\|\xi\|^{\kappa+1}\right), \quad \xi \rightarrow 0, \ell=1, \ldots, L .
$$

Moreover,

$$
\overline{\hat{\phi}}^{T}(\xi) \hat{\tilde{\phi}}(\xi)=1+O\left(\|\xi\|^{\kappa+1}\right), \quad \xi \rightarrow 0 .
$$

Proof. Suppose that the associated multiframelet transform has $\kappa+1$ balancing order. By Theorem 4.1 items 1) and 2) of Theorem 4.1 hold.

Since (4.5) holds with $\gamma=0$ and $C=1$, we must have (4.6) with $C=1$. Now (4.1) becomes

$$
\hat{c}(\xi) \hat{y}(\xi) \overline{\hat{\tilde{a}}}^{T}{ }^{T}=\hat{c}\left(M^{T} \xi\right) \hat{y}\left(M^{T} \xi\right)+O\left(\|\xi\|^{\kappa+1}\right), \quad \xi \rightarrow 0 .
$$

Since $\overline{\hat{\tilde{\phi}}}^{T}{ }^{T} \overline{\hat{\tilde{a}}}(\xi)^{T}={\overline{\hat{\tilde{\phi}}\left(M^{T} \xi\right)}}^{T}$, by Proposition 4.2 and (4.12), we conclude that there exists a nonzero constant $\tilde{C}$ such that $\tilde{C} \hat{\hat{c}}(\xi) \hat{y}(\xi)=\overline{\hat{\tilde{\phi}}(\xi)}^{T}+O\left(\|\xi\|^{\kappa+1}\right)$ as $\xi \rightarrow 0$. That is, (4.9) holds with $\hat{\tilde{c}}(\xi):=\tilde{C} \hat{\hat{c}}(\xi)$. Therefore, item (1) holds. By (4.9), we see that

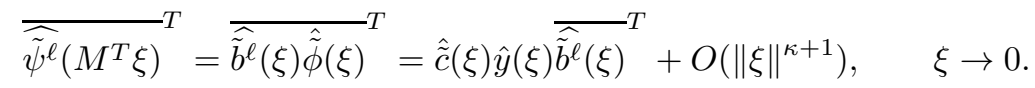

Consequently, by (4.2) and $\hat{\tilde{c}}(0) \neq 0$, (4.10) holds. Therefore, item (2) is verified.

Conversely, suppose that (4.9) and (4.10) are satisfied. Since $\hat{\tilde{c}}(0) \neq 0$, by Lemma 2.5 there is a $2 \pi$-periodic trigonometric polynomial $\hat{c}$ such that $\hat{c}(\xi)=$ $\hat{\tilde{c}}\left(M^{T} \xi\right) / \hat{\tilde{c}}(\xi)+O\left(\|\xi\|^{\kappa+1}\right)$ as $\xi \rightarrow 0$. By (4.9) and

$$
\overline{\hat{\tilde{\phi}}}(\xi)^{T} \overline{\tilde{\tilde{a}}}(\xi)^{T}={\overline{\hat{\tilde{\phi}}\left(M^{T} \xi\right)}}^{T},
$$

it is now straightforward to see that (4.1) is satisfied. By (4.9), (4.13) holds. By (4.10) and $\hat{\tilde{c}}(0) \neq 0$, it is straightforward to see that (4.2) holds. Now by Theorem 4.1, the multiframelet transform has $\kappa+1$ balancing order.

We now prove (4.11). By (4.5) with $\gamma=0$ and $C=1$, it follows from $\hat{\phi}\left(M^{T} \xi\right)=$ $\hat{a}(\xi) \hat{\phi}(\xi)$ that

$$
\begin{aligned}
\hat{c}\left(M^{T} \xi\right) \hat{y}\left(M^{T} \xi\right) \hat{\phi}\left(M^{T} \xi\right) & =\hat{c}\left(M^{T} \xi\right) \hat{y}\left(M^{T} \xi\right) \hat{a}(\xi) \hat{\phi}(\xi) \\
& =\hat{c}(\xi) \hat{y}(\xi) \hat{\phi}(\xi)+O\left(\|\xi\|^{\kappa+1}\right), \quad \xi \rightarrow 0 .
\end{aligned}
$$

Since $M$ is a dilation matrix, by [9, Lemma 2.2], we must have $\hat{c}(\xi) \hat{y}(\xi) \hat{\phi}(\xi)=$ $\hat{\grave{c}}(0) \hat{y}(0) \hat{\phi}(0)+O\left(\|\xi\|^{\kappa+1}\right)$ as $\xi \rightarrow 0$. As we proved, (4.9) holds with $\hat{\tilde{c}}(\xi)=\tilde{C} \hat{c}(\xi)$. Now we get

$$
\begin{aligned}
\overline{\hat{\phi}(\xi)}^{T} \hat{\tilde{\phi}}(\xi)= & \overline{\hat{\tilde{\phi}}}^{T}(\xi) \hat{\phi}(\xi)=\tilde{C} \hat{c}(\xi) \hat{y}(\xi) \hat{\phi}(\xi)+O\left(\|\xi\|^{\kappa+1}\right) \\
= & \tilde{C} \hat{c}(0) \hat{y}(0) \hat{\phi}(0)+O\left(\|\xi\|^{\kappa+1}\right), \quad \xi \rightarrow 0 .
\end{aligned}
$$

Since $\overline{\hat{\phi}}^{T}(0) \hat{\tilde{\phi}}(0)=1$, we now see that (4.11) holds. 
The other choice is to fix a mask $\hat{a}$ and all other filters $\widehat{b^{1}}, \ldots, \widehat{b^{L}}, \hat{\tilde{a}}, \widehat{\tilde{b^{1}}}, \ldots, \widehat{\tilde{b}^{L}}$. Therefore, the space $\mathcal{P}_{\kappa, y}$ is generally determined by $\hat{a}$. More precisely, the mask $\hat{a}$ is required to have $\kappa+1$ sum rules with a vector sequence $y \in\left(\ell_{0}\left(\mathbb{Z}^{d}\right)\right)^{1 \times r}$ in (2.2) (or (4.5) with $C=1$ ). Consequently, $y$ is known and the vector polynomial space $\mathcal{P}_{\kappa, y}$ is fixed by the given mask $\hat{a}$. By Theorem 4.1, prefiltering a given multiwavelet (e.g., see [14, 15]) or a multiframelet corresponds to designing a vector conversion operator $E$ such that $E\left(\Pi_{\kappa}\right)=\mathcal{P}_{\kappa, y}$. More precisely, we have the following result.

Proposition 4.4. Let $M$ be a $d \times d$ dilation matrix. Let $\hat{a}, \widehat{b^{1}}, \ldots, \widehat{b^{L}}$ and $\hat{\tilde{a}}, \widehat{\tilde{b}^{1}}, \ldots$, ${\widehat{\tilde{b}^{L}}}^{\mathrm{b}}$ be $r \times r$ matrices of $2 \pi$-periodic trigonometric polynomials in $d$ variables such that (1.9) holds. Suppose that the mask $\hat{a}$ satisfies the sum rules of order $\kappa+1$ with a vector sequence $y \in\left(\ell_{0}\left(\mathbb{Z}^{d}\right)\right)^{1 \times r}$ in (2.2). In order to prefilter the multiframelet transform so that it has $\kappa+1$ balancing order,

(1) one has to design a vector conversion operator $E$ satisfying $E\left(\Pi_{\kappa}\right)=\mathcal{P}_{\kappa, y}$. In particular, we can choose $E=C_{U} \circ E_{r}$ in Theorem 2.4, where $E_{r}$ is the standard vector conversion operator in (1.22) and $\hat{U}$ is a strongly invertible $r \times r$ matrix of $2 \pi$-periodic trigonometric polynomials such that (2.15) holds.

(2) The two conditions in (4.1) and (4.2) of Theorem 4.1 are satisfied.

In particular, if $L=|\operatorname{det} M|-1$ (this is true for a biorthogonal multiwavelet), then item (1) implies item (2) and item (2) can be dropped. Moreover, if the dual multiframelet $\hat{a}, \widehat{b^{1}}, \ldots, \widehat{b^{L}}, \hat{\tilde{a}}, \widehat{\tilde{b}^{1}}, \ldots, \widehat{\tilde{b}^{L}}$ can be prefiltered to have $\kappa+1$ balancing order (i.e., items (1) and (2) above hold), define

$$
\begin{aligned}
& \hat{\hat{a}}(\xi):=\hat{U}\left(M^{T} \xi\right) \hat{a}(\xi) \hat{U}(\xi)^{-1}, \quad \hat{\tilde{a}}(\xi):={\overline{\hat{U}}\left(M^{T} \xi\right)^{T}}^{-1} \hat{\tilde{a}}(\xi) \overline{\hat{U}(\xi)}^{T}, \\
& \widehat{\hat{b}^{\ell}}(\xi):=\widehat{b}^{\ell}(\xi) \hat{U}(\xi)^{-1}, \quad \widehat{\tilde{b}}^{\ell}(\xi):=\widehat{\widetilde{b}}^{\ell}(\xi) \overline{\hat{U}}(\xi)^{T}, \quad \ell=1, \ldots, L .
\end{aligned}
$$

Then $\hat{a}, \widehat{b^{1}}, \ldots, \widehat{b^{L}}$ and $\hat{\stackrel{a}{a}}, \widehat{\tilde{b}^{1}}, \ldots, \widehat{\tilde{b}^{L}}$ satisfy

$$
{\overline{P_{\left[\hat{\tilde{a}}, \widehat{\tilde{b}^{1}}, \ldots, \widehat{\tilde{b}}^{L}\right]}(\xi)}}^{T} P_{\left[\hat{a}, \widehat{b^{1}}, \ldots, \widehat{b}^{L}\right]}(\xi)=I_{m r} \quad \text { with } \quad m:=|\operatorname{det} M|,
$$

and their associated new dual multiframelet transform has $\kappa+1$ balancing order with respect to the standard vector conversion operator $E_{r}$. That is, the new dual multiframelet is balanced with $\kappa+1$ balancing order.

Proof. When $L=|\operatorname{det} M|-1$, since $\hat{a}$ satisfies the sum rules of order $\kappa+1$ with $y$ in (2.2), we have $S_{a, M} \mathcal{P}_{\kappa, y}=\mathcal{P}_{\kappa, y}$. Now by Theorem 4.1 and $E\left(\Pi_{\kappa}\right)=\mathcal{P}_{\kappa, y}$, the multiframelet transform has $\kappa+1$ balancing order with respect to the vector conversion operator $E$. Therefore, by Theorem 4.1. item (1) implies item (2). Hence, item (2) is not needed when $L=|\operatorname{det} M|-1$.

The identity (4.15) can be easily verified by (1.9) and (4.14). Let $\hat{Y}$ be defined in (2.9) such that $E_{r}\left(\Pi_{\kappa}\right)=\mathcal{P}_{\kappa, Y}$. We deduce from (2.15) and (4.1) that as $\xi \rightarrow 0$,

$$
\begin{aligned}
\hat{Y}(\xi) \overline{\hat{\tilde{a}}}^{T}(\xi) & =\hat{Y}(\xi) \hat{U}(\xi) \overline{\hat{\tilde{a}}(\xi)}^{T} \hat{U}\left(M^{T} \xi\right)^{-1}=\hat{y}(\xi) \overline{\tilde{\tilde{a}}}(\xi)^{T} \hat{U}\left(M^{T} \xi\right)^{-1}+O\left(\|\xi\|^{\kappa+1}\right) \\
& =\hat{c}(\xi) \hat{y}\left(M^{T} \xi\right) \hat{U}\left(M^{T} \xi\right)^{-1}+O\left(\|\xi\|^{\kappa+1}\right)=\hat{c}(\xi) \hat{Y}\left(M^{T} \xi\right)+O\left(\|\xi\|^{\kappa+1}\right) .
\end{aligned}
$$


By (2.15) and (4.2), we have

$$
\begin{aligned}
\hat{Y}(\xi) \overline{\hat{\tilde{b}}}^{T}(\xi)=\hat{Y}(\xi) \hat{U}(\xi) \overline{\tilde{\tilde{b}}}^{T}(\xi) & =\hat{y}(\xi){\overline{\tilde{b}^{\ell}}}^{T}{ }^{T}+O\left(\|\xi\|^{\kappa+1}\right) \\
& =O\left(\|\xi\|^{\kappa+1}\right), \quad \xi \rightarrow 0, \ell=1, \ldots, L .
\end{aligned}
$$

Hence, by Theorem 4.1, the new dual multiframelet has $\kappa+1$ balancing order with respect to the standard vector conversion operator $E_{r}$.

The convolution operator $C_{U}:\left(\ell\left(\mathbb{Z}^{d}\right)\right)^{1 \times r} \mapsto\left(\ell\left(\mathbb{Z}^{d}\right)\right)^{1 \times r}$ in item (1) of Proposition 4.4 is called the prefilter in the literature. The existence of such a prefilter $C_{U}$ and a vector conversion operator $E$ in Proposition 4.4 is guaranteed by Theorem 2.4. However, when $L \neq|\operatorname{det} M|-1$, the conditions in (4.1) and (4.2) are not automatically true and are not the direct consequence of (1.9) and the assumption that $\hat{a}$ has $\kappa+1$ sum rules with $y$ in (4.5). As a consequence, dual multiframelets generally have to be designed to satisfy (4.1) and (4.2). But when $L=|\operatorname{det} M|-1$, a dual multiframelet can always be prefiltered with the balancing order matching the order of the sum rules of $\hat{a}$. In particular, for biorthogonal multiwavelets, we have the following result.

Corollary 4.5. Let $M$ be $a d \times d$ dilation matrix and $L=|\operatorname{det} M|-1$. Let $\phi$ and $\tilde{\phi}$ be two compactly supported $r \times 1 M$-refinable function vectors in $L_{2}\left(\mathbb{R}^{d}\right)$ such

that (1.11) and (1.15) are satisfied. Suppose that $\hat{a}, \widehat{b^{1}}, \ldots, \widehat{b^{L}}$ and $\hat{\tilde{a}}, \widehat{\tilde{b}^{1}}, \ldots, \widehat{\tilde{b}^{L}}$ are $r \times r$ matrices of $2 \pi$-periodic trigonometric polynomials such that (1.9) holds. Define $\psi^{1}, \ldots, \psi^{L}$ and $\tilde{\psi}^{1}, \ldots \tilde{\psi}^{L}$ as in (1.12). If $\hat{a}$ has $\kappa+1$ sum rules with $y \in\left(\ell_{0}\left(\mathbb{Z}^{d}\right)\right)^{1 \times r}$ in (2.2), then $\left(\left\{\psi^{1}, \ldots, \psi^{L}\right\},\left\{\tilde{\psi}^{1}, \ldots, \tilde{\psi}^{L}\right\}\right)$ generates a pair of biorthogonal $M$ multiwavelets in $L_{2}\left(\mathbb{R}^{d}\right)$ with $\tilde{\psi}^{1}, \ldots, \tilde{\psi}^{m}$ having $\kappa+1$ vanishing moments. Let $E=$ $C_{U} \circ E_{r}$ as in item (1) of Proposition 4.4 and define $\hat{a}, \widehat{\hat{b}^{1}}, \ldots, \widehat{\hat{b}^{L}}$ and $\hat{\tilde{a}}, \widehat{\tilde{\tilde{b}}^{1}}, \ldots, \widehat{\tilde{\tilde{b}}^{L}}$ as in (4.14). Define $\dot{\phi}$ and $\stackrel{\tilde{\phi}}{\text { by }} \hat{\grave{\phi}}(\xi):=\hat{U}(\xi) \hat{\phi}(\xi)$ and $\hat{\tilde{\phi}}(\xi):={\overline{\hat{U}}(\xi)^{T}}^{-1} \hat{\tilde{\phi}}(\xi)$. Then

$$
\begin{aligned}
& \hat{\dot{\phi}}\left(M^{T} \xi\right)=\hat{\hat{a}}(\xi) \hat{\dot{\phi}}(\xi), \quad \hat{\grave{\phi}}\left(M^{T} \xi\right)=\hat{\tilde{\tilde{a}}}(\xi) \hat{\dot{\tilde{\phi}}}(\xi), \\
& \widehat{\psi^{\ell}}\left(M^{T} \xi\right)=\widehat{b^{\ell}}(\xi) \hat{\dot{\phi}}(\xi), \quad \widehat{\tilde{\psi}^{\ell}}\left(M^{T} \xi\right)=\widehat{\tilde{\tilde{b}}}(\xi) \hat{\tilde{\phi}}(\xi),
\end{aligned}
$$

the new multiframelet transform (more precisely, a biorthogonal multiwavelet transform) has $\kappa+1$ balancing order, and $\left(\left\{\psi^{1}, \ldots, \psi^{m}\right\},\left\{\tilde{\psi}^{1}, \ldots, \tilde{\psi}^{m}\right\}\right)$ generates a pair of balanced biorthogonal $M$-multiwavelets in $L_{2}\left(\mathbb{R}^{d}\right)$ with $\kappa+1$ balancing order using the new generators $\stackrel{\circ}{\phi}, \stackrel{\tilde{\phi}}{\hat{a}}, \hat{a}, \widehat{b^{1}}, \ldots, \widehat{b^{L}}, \hat{\tilde{a}}, \widehat{\tilde{\tilde{b}}^{1}}, \ldots, \widehat{\tilde{\tilde{b}}^{L}}$.

\section{A NEW CANONICAL FORM OF A MATRIX MASK}

In this section, we shall develop a canonical form of a multivariate matrix mask. As demonstrated in [9, 11, 13, the canonical form of a matrix mask greatly facilitates the study of refinable function vectors, vector subdivision schemes, and the construction of dual multiframelets. Following the lines developed in [11, 13, for the univariate setting, in this section we shall introduce a new canonical form of a multivariate matrix mask with multiplicity greater than one, by adding new features to the existing canonical form of a matrix mask in [9, 11, 13. The new canonical form of a matrix mask plays a critical role in the investigation and construction 
of balanced multivariate biorthogonal multiwavelets and balanced multiframelet transforms.

Recall that $\hat{U}$ is strongly invertible if both $\hat{U}(\xi)$ and $\hat{U}(\xi)^{-1}$ are matrices of $2 \pi$-periodic trigonometric polynomials. First, we prove Lemma 2.3

Proof of Lemma 2.3. We first prove the case $s=1$ by showing that there exists a strongly invertible $r \times r$ matrix $\hat{V}$ of $2 \pi$-periodic trigonometric polynomials such that

$$
\hat{y}(\xi) \hat{V}(\xi)=(1,0, \ldots, 0)+O\left(\|\xi\|^{n}\right), \quad \xi \rightarrow 0 .
$$

Denote $\left(\hat{y}_{1}(\xi), \ldots, \hat{y}_{r}(\xi)\right):=\hat{y}(\xi)$. Since $\hat{y}(0) \neq 0$, without loss of any generality, we may assume $\hat{y}_{1}(0) \neq 0$; otherwise, we perform a permutation on $\hat{y}$ first. Choose $2 \pi$-periodic trigonometric polynomials $\hat{c}_{1}, \hat{c}_{2}, \ldots, \hat{c}_{r}$ such that $\hat{c}_{1}(\xi)=1 / \hat{y}_{1}(\xi)+$ $O\left(\|\xi\|^{n}\right)$ and $\hat{c}_{\ell}(\xi)=\hat{y}_{\ell}(\xi) / \hat{y}_{1}(\xi)+O\left(\|\xi\|^{n}\right)$, as $\xi \rightarrow 0$, for $\ell=2, \ldots, r$. That is, we have

$$
\begin{aligned}
& \hat{c}_{1}(\xi) \hat{y}_{1}(\xi)=1+O\left(\|\xi\|^{n}\right) \quad \text { and } \\
& \hat{y}_{\ell}(\xi)-\hat{c}_{\ell}(\xi) \hat{y}_{1}(\xi)=O\left(\|\xi\|^{n}\right), \quad \xi \rightarrow 0, \ell=2, \ldots, r .
\end{aligned}
$$

Note that $\hat{c}_{1}(0) \neq 0$. Therefore, we have

$$
\begin{aligned}
\left(1-\hat{c}_{1}(\xi) / \hat{c}_{1}(0)\right)^{2 n}=1 & -\hat{c}_{1}(\xi) Q_{n}(\xi) \\
\text { with } \quad Q_{n}(\xi) & :=\sum_{k=0}^{2 n-1}(-1)^{k}\left(\begin{array}{c}
2 n \\
k+1
\end{array}\right)\left[\hat{c}_{1}(\xi)\right]^{k} /\left[\hat{c}_{1}(0)\right]^{k+1} .
\end{aligned}
$$

Define two strongly invertible matrices $\hat{U}_{1}$ and $\hat{U}_{2}$ by

$$
\hat{U}_{1}(\xi):=\left(\begin{array}{cc}
1 & -\hat{f}(\xi) \\
0 & I_{r-1}
\end{array}\right) \quad \text { with } \quad \hat{f}(\xi):=\left(\hat{c}_{2}(\xi), \ldots, \hat{c}_{r}(\xi)\right)
$$

and

$$
\hat{U}_{2}(\xi):=\left(\begin{array}{ccc}
\hat{c}_{1}(\xi) & -\left(1-\hat{c}_{1}(\xi) / \hat{c}_{1}(0)\right)^{n} & 0 \\
\left(1-\hat{c}_{1}(\xi) / \hat{c}_{1}(0)\right)^{n} & Q_{n}(\xi) & 0 \\
0 & 0 & I_{r-2}
\end{array}\right) .
$$

Note that $\operatorname{det} \hat{U}_{1}(\xi)=\operatorname{det} \hat{U}_{2}(\xi)=1$ by (5.3). Therefore, $\hat{U}_{1}$ and $\hat{U}_{2}$ are strongly invertible. Now by (5.2) and (5.3), it is easy to check (5.1) by taking $\hat{V}(\xi):=$ $\hat{U}_{1}(\xi) \hat{U}_{2}(\xi)$. In fact, by (5.2), as $\xi \rightarrow 0$, we have

$$
\begin{aligned}
\hat{y}(\xi) \hat{U}_{1}(\xi) \hat{U}_{2}(\xi) & =\left(\hat{y}_{1}(\xi), 0, \ldots, 0\right) \hat{U}_{2}(\xi)+O\left(\|\xi\|^{n}\right) \\
& =\left(\hat{c}_{1}(\xi) \hat{y}_{1}(\xi), 0, \ldots, 0\right)+O\left(\|\xi\|^{n}\right)=(1,0, \ldots, 0)+O\left(\|\xi\|^{n}\right) .
\end{aligned}
$$

Similarly, there exists a strongly invertible $r \times r$ matrix $\hat{\tilde{V}}$ of $2 \pi$-periodic trigonometric polynomials such that $\hat{\tilde{y}}(\xi) \hat{\tilde{V}}(\xi)=(1,0, \ldots, 0)+O\left(\|\xi\|^{n}\right)$ as $\xi \rightarrow 0$. Now the proof for the case $s=1$ is completed by taking $\hat{U}(\xi):=\hat{\tilde{V}}(\xi)[\hat{V}(\xi)]^{-1}$.

We now prove the general claim. Clearly, it suffices to show the claim with $\hat{y}(\xi)=\left(I_{s}, 0\right)$. We now prove it by induction on $s$ with $s<r$. As we just proved, the claim holds for $s=1$. Suppose that the claim holds for $s-1$ with $s<r$. Now we prove it for the case $s$. Let $\hat{c}$ denote the first row of the matrix $\hat{\tilde{y}}$. Since $\hat{\tilde{y}}(0)$ 
has the full rank $s$, we must have $\hat{c}(0) \neq 0$. Now by what has been proved, there is a strongly invertible matrix $\hat{U}_{1}$ such that

$$
\hat{\tilde{y}}(\xi) \hat{U}_{1}(\xi)=\left(\begin{array}{cc}
1+O\left(\|\xi\|^{\kappa+1}\right) & O\left(\|\xi\|^{\kappa+1}\right) \\
* & \hat{y}(\xi)
\end{array}\right), \quad \xi \rightarrow 0,
$$

where $*$ denotes some column vector of $2 \pi$-periodic trigonometric polynomials and $\hat{y}$ is an $(s-1) \times(r-1)$ matrix of $2 \pi$-periodic trigonometric polynomials. Since $\hat{U}_{1}$ is strongly invertible and $\hat{\tilde{y}}(0)$ has full rank $s$, we can deduce that $\hat{y}(0)$ has the full rank $s-1$. Since $s-1<r-1$, by the induction hypothesis, there exists a strongly invertible $(r-1) \times(r-1)$ matrix $\hat{V}(\xi)$ such that

$$
\hat{\grave{y}}(\xi) \hat{V}(\xi)=\left(I_{s-1}, 0\right)+O\left(\|\xi\|^{\kappa+1}\right), \quad \xi \rightarrow 0 .
$$

Denote $\hat{U}_{2}(\xi):=\operatorname{diag}(1, \hat{V}(\xi))$. Then we observe that

$$
\hat{\tilde{y}}(\xi) \hat{U}_{1}(\xi) \hat{U}_{2}(\xi)=\left(\hat{U}_{3}(\xi), 0\right)+O\left(\|\xi\|^{\kappa+1}\right), \quad \xi \rightarrow 0,
$$

where $\hat{U}_{3}(\xi)$ is an $s \times s$ lower-triangular matrix with all ones in its diagonal and with all other lower-triangular elements being $2 \pi$-periodic trigonometric polynomials. Consequently, the matrix $\hat{U}_{3}$ is strongly invertible. Define

$$
\hat{U}(\xi):=\hat{U}_{1}(\xi) \hat{U}_{2}(\xi) \operatorname{diag}\left(\hat{U}_{3}(\xi)^{-1}, I_{r-s}\right) .
$$

By calculation, it is not difficult to see that (2.12) holds with $\hat{y}(\xi)=\left(I_{s}, 0\right)$.

Let $\phi$ be an $M$-refinable function (or distribution) vector satisfying $\hat{\phi}\left(M^{T} \xi\right)=$ $\hat{a}(\xi) \hat{\phi}(\xi)$. For a strongly invertible matrix $\hat{U}$, we define

$$
\hat{a}(\xi):=\hat{U}\left(M^{T} \xi\right) \hat{a}(\xi) \hat{U}(\xi)^{-1} \quad \text { and } \quad \hat{\phi}(\xi):=\hat{U}(\xi) \hat{\phi}(\xi) .
$$

Then we have $\hat{\dot{\phi}}\left(M^{T} \xi\right)=\hat{\grave{a}}(\xi) \hat{\grave{\phi}}(\xi)$. It is evident that $\phi$ is compactly supported if and only if $\dot{\phi}$ is compactly supported. Similarly, $a$ is finitely supported if and only if $\stackrel{\circ}{a}$ is finitely supported.

Now we have the following result on the canonical form of a multivariate matrix mask.

Theorem 5.1. Let $M$ be a $d \times d$ dilation matrix. Let $\hat{a}$ be an $r \times r$ matrix of $2 \pi$ periodic trigonometric polynomials in $d$ variables with multiplicity $r>1$. Suppose that $\hat{a}$ satisfies the sum rules of order $\kappa+1$ in (2.2) with the dilation matrix $M$ and $a 1 \times r$ vector $\hat{y}$ of $2 \pi$-periodic trigonometric polynomials. Then there exists a strongly invertible $r \times r$ matrix $\hat{U}$ (that is, both $\hat{U}$ and $\hat{U}^{-1}$ are matrices of $2 \pi$ periodic trigonometric polynomials) such that

$$
\hat{a}(\xi):=\left(\begin{array}{ll}
\hat{a}_{1,1}(\xi) & \hat{a}_{1,2}(\xi) \\
\hat{a}_{2,1}(\xi) & \hat{a}_{2,2}(\xi)
\end{array}\right):=\hat{U}\left(M^{T} \xi\right) \hat{a}(\xi) \hat{U}(\xi)^{-1}
$$

has the following property:

$$
\begin{aligned}
& \hat{\grave{a}}_{1,1}(\xi+2 \pi \gamma)=\delta_{\gamma}+O\left(\|\xi\|^{\kappa+1}\right) \text { and } \\
& \hat{\grave{a}}_{1,2}(\xi+2 \pi \gamma)=O\left(\|\xi\|^{\kappa+1}\right), \quad \xi \rightarrow 0, \gamma \in \Gamma_{M^{T}} \text {, }
\end{aligned}
$$

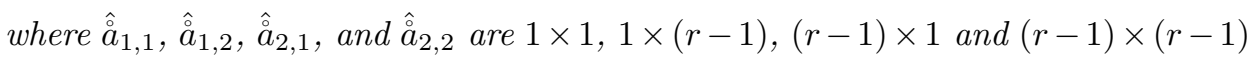
matrices of $2 \pi$-periodic trigonometric polynomials. If in addition there is an $r \times 1$ vector $\phi$ of compactly supported tempered distributions satisfying

$$
\hat{\phi}\left(M^{T} \xi\right)=\hat{a}(\xi) \hat{\phi}(\xi), \quad \xi \in \mathbb{R}^{d} \quad \text { and } \quad \hat{y}(0) \hat{\phi}(0) \neq 0,
$$


then for any nonnegative integer $n$, there is a strongly invertible matrix $\hat{U}$ such that (5.6) and (5.7) are satisfied with the additional properties:

$$
\hat{\grave{a}}_{1,1}(\xi)=1+O\left(\|\xi\|^{n}\right), \quad \hat{\grave{a}}_{2,1}(\xi)=O\left(\|\xi\|^{n}\right), \quad \xi \rightarrow 0
$$

and

$$
\widehat{\dot{\phi}}_{1}(\xi)=1+O\left(\|\xi\|^{n}\right) \quad \text { and } \quad \widehat{\dot{\phi}}_{\ell}(\xi)=O\left(\|\xi\|^{n}\right), \quad \xi \rightarrow 0, \ell=2, \ldots, r,
$$

where $\hat{\dot{\phi}}(\xi):=\left(\widehat{\dot{\phi}_{1}}, \ldots, \widehat{\dot{\phi}_{r}}\right)^{T}:=\hat{U}(\xi) \hat{\phi}(\xi)$. Thus, except for $\stackrel{\circ}{\phi}_{1}$, all other components $\stackrel{\circ}{\phi}_{2}, \ldots, \dot{\phi}_{r}$ have $n$ vanishing moments.

Proof. Since $\hat{y}(0) \neq 0$, by Lemma 2.3 , there is a strongly invertible matrix $\hat{U}$ such that

That is,

$$
\hat{y}(\xi) \hat{U}(\xi)^{-1}=(1,0, \ldots, 0)+O\left(\|\xi\|^{\kappa+1}\right), \quad \xi \rightarrow 0 .
$$

$$
(1,0, \ldots, 0)=\hat{y}\left(M^{T} \xi\right) \hat{U}\left(M^{T} \xi\right)+O\left(\|\xi\|^{\kappa+1}\right), \quad \xi \rightarrow 0 .
$$

Since $\hat{a}$ satisfies the sum rules of order $\kappa+1$ in (2.2), we deduce that for $\gamma \in \Gamma_{M^{T}}$, as $\xi \rightarrow 0$,

$$
\begin{aligned}
(1,0, \ldots, 0) \hat{a} & (\xi+2 \pi \gamma) \\
& =\hat{y}\left(M^{T} \xi\right) \hat{U}\left(M^{T} \xi\right)^{-1} \hat{U}\left(M^{T} \xi\right) \hat{a}(\xi+2 \pi \gamma) \hat{U}(\xi+2 \pi \gamma)^{-1}+O\left(\|\xi\|^{\kappa+1}\right) \\
& =y\left(M^{T} \xi\right) \hat{a}(\xi+2 \pi \gamma) \hat{U}(\xi+2 \pi \gamma)^{-1}+O\left(\|\xi\|^{\kappa+1}\right) \\
& =\delta_{\gamma} \hat{y}(\xi) \hat{U}(\xi+2 \pi \gamma)^{-1}+O\left(\|\xi\|^{\kappa+1}\right) \\
& =\left(\delta_{\gamma}, 0, \ldots, 0\right)+O\left(\|\xi\|^{\kappa+1}\right) .
\end{aligned}
$$

That is, we have

$$
(1,0, \ldots, 0) \hat{a}(\xi+2 \pi \gamma)=\left(\delta_{\gamma}, 0, \ldots, 0\right)+O\left(\|\xi\|^{\kappa+1}\right), \quad \xi \rightarrow 0, \gamma \in \Gamma_{M^{T}}
$$

In other words, $\hat{a}$ satisfies the sum rules of order $\kappa$ with the vector $\hat{y}$ in (2.2) being $[1,0, \ldots, 0]$. Now it is straightforward to see that (5.7) is equivalent to the relation in (5.11).

In addition, suppose that (5.8) is satisfied. By what has been proved, there exists a strongly invertible matrix $\hat{\tilde{U}}$ such that

$$
\hat{y}(\xi) \hat{\tilde{U}}(\xi)^{-1}=(1,0, \ldots, 0)+O\left(\|\xi\|^{\kappa+1}\right), \quad \xi \rightarrow 0
$$

and

satisfies

$$
\hat{\tilde{a}}(\xi):=\left(\begin{array}{ll}
\hat{\tilde{a}}_{1,1}(\xi) & \hat{\tilde{a}}_{1,2}(\xi) \\
\tilde{\tilde{a}}_{2,1}(\xi) & \tilde{\tilde{a}}_{2,2}(\xi)
\end{array}\right):=\hat{\tilde{U}}\left(M^{T} \xi\right) \hat{a}(\xi) \hat{\tilde{U}}(\xi)^{-1}
$$

$$
\begin{aligned}
& \hat{\tilde{a}}_{1,1}(\xi+2 \pi \gamma)=\delta_{\gamma}+O\left(\|\xi\|^{\kappa+1}\right) \quad \text { and } \\
& \hat{\tilde{a}}_{1,2}(\xi+2 \pi \gamma)=O\left(\|\xi\|^{\kappa+1}\right), \quad \xi \rightarrow 0, \gamma \in \Gamma_{M^{T}}
\end{aligned}
$$

Denote

$$
\hat{\tilde{\phi}}(\xi):=\left(\hat{\tilde{\phi}}_{1}(\xi), \ldots, \hat{\tilde{\phi}}_{r}(\xi)\right)^{T}:=\hat{\tilde{U}}(\xi) \hat{\phi}(\xi) .
$$

Clearly, by (5.8) and (5.12), we have

$$
\hat{\tilde{\phi}}_{1}(0)=(1,0, \ldots, 0) \hat{\tilde{\phi}}(0)=\hat{y}(0) \hat{\tilde{U}}(0)^{-1} \hat{\tilde{U}}(0) \hat{\phi}(0)=\hat{y}(0) \hat{\phi}(0) \neq 0 .
$$


Without loss of any generality, we can assume that $n>\kappa$. Since $\hat{\tilde{\phi}}_{1}(0) \neq 0$, as in the proof of Lemma 2.3, we can choose $2 \pi$-periodic trigonometric polynomials $\hat{c}_{1}, \ldots, \hat{c}_{r}$ such that

$$
\begin{aligned}
& \hat{c}_{1}(\xi) \hat{\tilde{\phi}}_{1}(\xi)=1+O\left(\|\xi\|^{n}\right) \quad \text { and } \\
& \hat{\tilde{\phi}}_{\ell}(\xi)-\hat{c}_{\ell}(\xi) \hat{\tilde{\phi}}_{1}(\xi)=O\left(\|\xi\|^{n}\right), \quad \xi \rightarrow 0, \ell=2, \ldots, r .
\end{aligned}
$$

Define two strongly invertible matrices $\hat{U}_{1}$ and $\hat{U}_{2}$ as in (5.4) and (5.5). Then we take a strongly invertible matrix $\hat{U}(\xi):=\left[\hat{U}_{1}(\xi) \hat{U}_{2}(\xi)\right]^{T} \hat{\tilde{U}}(\xi)$. We now show that $\hat{U}$ is a desired strongly invertible matrix such that all the claims in Theorem 5.1 hold. By (5.12), as $\xi \rightarrow 0$, we have

$$
\begin{aligned}
\hat{y}(\xi) \hat{U}(\xi)^{-1} & =\hat{y}(\xi) \hat{\tilde{U}}(\xi)^{-1}\left[\hat{U}_{2}(\xi)^{T} \hat{U}_{1}(\xi)^{T}\right]^{-1} \\
& =(1,0, \ldots, 0)\left(\hat{U}_{1}(\xi)^{-1}\right)^{T}\left(\hat{U}_{2}(\xi)^{-1}\right)^{T}+O\left(\|\xi\|^{\kappa+1}\right) .
\end{aligned}
$$

By the choice of $\hat{U}_{1}$ in (5.4) and $\hat{U}_{2}$ in (5.5), since we assumed that $n \geqslant \kappa$, we see that

and

$$
(1,0, \ldots, 0)\left[\hat{U}_{1}(\xi)^{-1}\right]^{T}=(1,0, \ldots, 0)
$$

$$
(1,0, \ldots, 0)\left[\hat{U}_{2}(\xi)^{-1}\right]^{T}=\left(Q_{n}(\xi),\left(1-\hat{c}_{1}(\xi) / \hat{c}_{1}(0)\right)^{n}, 0, \ldots, 0\right) .
$$

That is, by (5.16), as $\xi \rightarrow 0$, we deduce that

$$
\begin{aligned}
\hat{y}(\xi) \hat{U}(\xi)^{-1} & =\left(Q_{n}(\xi),\left(1-\hat{c}_{1}(\xi) / \hat{c}_{1}(0)\right)^{n}, 0, \ldots, 0\right)+O\left(\|\xi\|^{\kappa+1}\right) \\
& =\left(Q_{n}(\xi), 0, \ldots, 0\right)+O\left(\|\xi\|^{\kappa+1}\right) .
\end{aligned}
$$

By (5.3) and (5.15), we see that as $\xi \rightarrow 0$,

$$
\begin{aligned}
Q_{n}(\xi) & =1 / \hat{c}_{1}(\xi)+\left(1-\hat{c}_{1}(\xi) / \hat{c}_{1}(0)\right)^{2 n} / \hat{c}_{1}(\xi)=1 / \hat{c}_{1}(\xi)+O\left(\|\xi\|^{2 n}\right) \\
& =\hat{c}_{1}(\xi) \widetilde{\tilde{\phi}}_{1}(\xi) / \hat{c}_{1}(\xi)+O\left(\|\xi\|^{n}\right)=\widehat{\tilde{\phi}}_{1}(\xi)+O\left(\|\xi\|^{n}\right) .
\end{aligned}
$$

Since $\hat{\tilde{\phi}}\left(M^{T} \xi\right)=\hat{\tilde{a}}(\xi) \hat{\tilde{\phi}}(\xi)$, now by (5.13) we have $\hat{\tilde{\phi}}_{1}\left(M^{T} \xi\right)=\hat{\tilde{\phi}}_{1}(\xi)+O\left(\|\xi\|^{\kappa}\right)$ as $\xi \rightarrow 0$. Since $M$ is a dilation matrix, it now follows from this relation that $\hat{\tilde{\phi}}_{1}(\xi)=\hat{\tilde{\phi}}_{1}(0)+O\left(\|\xi\|^{\kappa}\right)$ as $\xi \rightarrow 0$ (see [9, Lemma 2.2]). That is, by (5.17), we have

$$
\hat{y}(\xi) \hat{U}(\xi)^{-1}=\left(\widehat{\tilde{\phi}}_{1}(0), 0, \ldots, 0\right)+O\left(\|\xi\|^{\kappa+1}\right), \quad \xi \rightarrow 0 .
$$

Therefore, by $\widehat{\tilde{\phi}}_{1}(0) \neq 0$ and the same argument as in the first part of the proof, (5.7) is satisfied. More precisely, we see that $\stackrel{\circ}{a}$ satisfies the sum rules of order $\kappa$ with the sequence $\left(\widehat{\tilde{\phi}_{1}}(0), 0, \ldots, 0\right)$, from which we easily deduce that (5.7) holds.

By (5.15) and (5.3), we have

$$
\begin{aligned}
& \hat{\grave{\phi}}(\xi)=\hat{U}_{2}(\xi)^{T} \hat{U}_{1}(\xi)^{T} \hat{\tilde{U}}(\xi) \hat{\phi}(\xi)=\left[\hat{\tilde{\phi}}(\xi)^{T} \hat{U}_{1}(\xi) \hat{U}_{2}(\xi)\right]^{T} \\
& =(1,0, \ldots, 0)^{T}+O\left(\|\xi\|^{n}\right), \quad \xi \rightarrow 0 .
\end{aligned}
$$

That is, (5.10) holds. To prove (5.9), we can easily verify that $\hat{\grave{\phi}}\left(M^{T} \xi\right)=\hat{a}(\xi) \hat{\dot{\phi}}(\xi)$. Therefore, by (5.10), we deduce that

$$
\hat{\grave{\phi}}\left(M^{T} \xi\right)=\hat{\hat{a}}_{1,1}(\xi) \hat{\grave{\phi}}_{1}(\xi)+O\left(\|\xi\|^{n}\right) \quad \text { and } \quad \hat{\hat{a}}_{1,2}(\xi) \hat{\grave{\phi}}_{1}(\xi)=O\left(\|\xi\|^{n}\right), \quad \xi \rightarrow 0 .
$$

Now it follows easily from (5.10) that (5.9) holds. 
To complete this section, following the lines developed in [11, 13], we shortly sketch the construction procedure of dual multiframelets with high balancing orders. Let $\hat{a}$ and $\hat{\tilde{a}}$ be given $r \times r$ matrices of $2 \pi$-periodic trigonometric polynomials such that $\hat{a}$ has $\kappa+1$ sum rules in (2.2) with $y$ and $\hat{\tilde{a}}$ has $\tilde{\kappa}+1$ sum rules with $\tilde{y}$. Let $\hat{U}$ and $\hat{\tilde{U}}$ be given in Theorem [5.1 such that $\hat{a}(\xi):=\hat{U}\left(M^{T} \xi\right) \hat{a}(\xi) \hat{U}(\xi)^{-1}$ and $\hat{\tilde{a}}(\xi):=$ $\hat{\tilde{U}}\left(M^{T} \xi\right) \hat{\tilde{a}}(\xi) \hat{\tilde{U}}(\xi)^{-1}$ have the desirable properties as described by Theorem 5.1 (for the part of $\tilde{a}$, replace $a$ and $U$ by $\tilde{a}$ and $\tilde{U})$. Now one constructs $r \times r$ matrices $\widehat{b^{1}}, \ldots, \widehat{\widehat{b}^{L}}$ and $\widehat{\tilde{\tilde{b}}^{1}}, \ldots, \widehat{\tilde{\tilde{b}}^{L}}$ such that (4.15) holds and

$$
\begin{aligned}
& (1,0, \ldots, 0){\overline{\hat{\hat{b}^{\ell}}}}^{T}(\xi)=O\left(\|\xi\|^{\tilde{\kappa}+1}\right),
\end{aligned}
$$

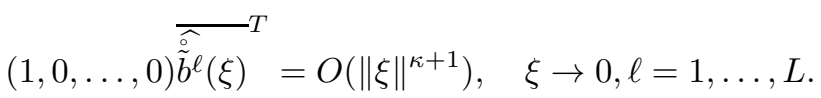

Then the dual multiframelet transform associated with $\hat{a}, \widehat{b^{1}}, \ldots, \widehat{\widehat{b}^{L}}$ and $\hat{\tilde{a}}, \widehat{\tilde{\tilde{b}^{1}}}$, $\ldots, \widehat{\tilde{b}^{L}}$ has $\kappa+1$ balancing order. One can also equivalently transform back to $\hat{a}$ (or $\hat{\tilde{a}}$ ) from $\hat{a}$ (or $\hat{\tilde{a}}$ ) by using $\hat{U}$ (or $\hat{\tilde{U}}$ ). For more details, see [1] for the univariate case.

\section{Connections to BALANCing ORDERS OF Multiwavelets IN THE LITERATURE}

For the convenience of the reader, in this section we shall connect our notion and results on balancing orders of multiframelets in the discrete data setting with other notions of balancing order in the literature for biorthogonal multiwavelets in the function setting.

In the univariate setting, for a compactly supported orthonormal 2-refinable function vector $\tilde{\phi}=\left(\tilde{\phi}_{1}, \ldots, \tilde{\phi}_{r}\right)^{T}$ in $L_{2}(\mathbb{R})$, a notion of $\kappa+1$ balancing order for $\phi$ is introduced in [18, 19] saying that

$$
\begin{array}{r}
\int_{\mathbb{R}} \tilde{\phi}_{1}(x) x^{j} d x=\int_{\mathbb{R}} \tilde{\phi}_{2}(x)\left(x-\frac{1}{r}\right)^{j} d x=\cdots=\int_{\mathbb{R}} \tilde{\phi}_{r}(x)\left(x-\frac{r-1}{r}\right)^{j} d x, \\
j=0, \ldots, \kappa .
\end{array}
$$

This notion was further generalized in $[3]$ to multivariate biorthogonal multiwavelets. For a compactly supported $M$-refinable function vector $\tilde{\phi}=\left(\tilde{\phi}_{1}, \ldots, \tilde{\phi}_{r}\right)^{T}$ in $L_{2}\left(\mathbb{R}^{d}\right)$ and some points $\alpha_{0}, \ldots, \alpha_{r-1} \in \mathbb{R}^{d}, \tilde{\phi}$ is said to have $\kappa+1$ balancing order (see [3, (3.1)]) relative to the $r$-tuple $\left[\alpha_{0}, \ldots, \alpha_{r-1}\right]$ if

$$
\begin{aligned}
\int_{\mathbb{R}^{d}} \tilde{\phi}_{1}(x)\left(x-\alpha_{0}\right)^{\mu} d x & =\int_{\mathbb{R}^{d}} \tilde{\phi}_{2}(x)\left(x-\alpha_{1}\right)^{\mu} d x \\
& =\cdots=\int_{\mathbb{R}^{d}} \tilde{\phi}_{r}(x)\left(x-\alpha_{r-1}\right)^{\mu} d x, \quad|\mu| \leqslant \kappa .
\end{aligned}
$$

Denote $\omega_{j}:=N \alpha_{j}$ for $j=0, \ldots, r-1$ and $\hat{Y}(\xi):=\left(e^{i N^{-1} \omega_{0} \cdot \xi}, \ldots, e^{i N^{-1} \omega_{r-1} \cdot \xi}\right)$ as in (2.9) of Proposition 2.2. In the frequency domain, noting that $\int_{\mathbb{R}^{d}} \tilde{\phi}_{j}(x)(x-$ $\left.\alpha_{j-1}\right)^{\mu} d x=i^{|\mu|} \partial^{\mu}\left[e^{i \xi \cdot \alpha_{j-1}} \widetilde{\tilde{\phi}}_{j}(\xi)\right](0)$ (derivative on the variable $\xi$ only), we see that 
(6.2) is equivalent to

$$
\overline{\hat{\tilde{\phi}}}^{T}(\xi)=\hat{\tilde{c}}(\xi) \hat{Y}(\xi)+O\left(\|\xi\|^{\kappa+1}\right), \quad \xi \rightarrow 0
$$

for some $2 \pi$-periodic trigonometric polynomial $\hat{\tilde{c}}$. We observed that 3 uses the mapping $\stackrel{\circ}{E}: h \in \Pi_{\kappa} \mapsto\left(h\left(\cdot+\alpha_{1}\right), \ldots, h\left(\cdot+\alpha_{r-1}\right)\right) \in \Pi_{\kappa}^{1 \times r}$; however, for general $r$-tuples $\left[\alpha_{0}, \ldots, \alpha_{r-1}\right]$, the existence and construction of such a vector conversion operator are not known in 3. By Theorem 2.4, we have a desired vector conversion operator $E: \ell\left(\mathbb{Z}^{d}\right) \mapsto\left(\ell\left(\mathbb{Z}^{d}\right)\right)^{1 \times r}$ such that $\mathcal{P}_{\kappa, Y}=E\left(\Pi_{\kappa}\right)=\stackrel{\circ}{E}\left(\Pi_{\kappa}\right)$, since by (2.10) and $\alpha_{j}=N^{-1} \omega_{j}$,

$$
\begin{aligned}
E\left(h\left(N^{-1} \cdot\right)\right) & =\left(h\left(N^{-1}\left(N \cdot+\omega_{0}\right)\right), \ldots, h\left(N^{-1}\left(N \cdot+\omega_{r-1}\right)\right)\right) \\
& =\left(h\left(\cdot+\alpha_{0}\right), \ldots, h\left(\cdot+\alpha_{r-1}\right)\right), \quad h \in \Pi_{\kappa} .
\end{aligned}
$$

We now show that our notion of balancing order with respect to a vector conversion operator $E$ satisfying $E\left(\Pi_{\kappa}\right)=\mathcal{P}_{\kappa, Y}$ agrees with the one in (6.2), or equivalently (6.3), for biorthogonal multiwavelets.

Assume that there are compactly supported $1 \times r$ function vectors $\phi, \psi^{1}, \ldots, \psi^{L}$, $\tilde{\phi}, \tilde{\psi}^{1}, \ldots, \tilde{\psi}^{L}$ in $L_{2}\left(\mathbb{R}^{d}\right)$ such that (1.11) and (1.12) are satisfied. As in [3, 4, 18, 19], for the case of biorthogonal multiwavelets, we also assume that the biorthogonality condition in (1.15) holds and the mask $\hat{a}$ has $\kappa+1$ sum rules with $y \in\left(\ell_{0}\left(\mathbb{Z}^{d}\right)\right)^{1 \times r}$ in (2.2) (that is, (3.17) holds with $C=1$ and $\hat{c} \equiv 1$ ). By the biorthogonality relation in (1.15), we deduce that $\phi$ and $\tilde{\phi}$ are compactly supported $M$-refinable function vectors in $L_{2}\left(\mathbb{R}^{d}\right)$ with stable integer shifts. Consequently, it is known (see [9, Proposition 3.1]) that the condition in item (2) of Proposition 4.2 holds for all $\kappa \in \mathbb{N}$ for both $\hat{\tilde{a}}(0)$ and $\hat{a}(0)$, since 1 is a simple eigenvalue of $\hat{a}(0)$ and $\hat{\tilde{a}}(0)$ and all the other eigenvalues of $\hat{a}(0)$ and $\hat{\tilde{a}}(0)$ are less than one in modulus. Since $\hat{a}$ has $\kappa+1$ sum rules in (2.2) with a vector sequence $y \in\left(\ell_{0}\left(\mathbb{Z}^{d}\right)\right)^{1 \times r}$ and $L=|\operatorname{det} M|-1$, using (1.9) and (2.2), we can easily check that (4.10) must hold and (cf. [9, Proposition 2.2])

$$
\hat{y}(\xi) \hat{\phi}(\xi+2 \pi k)=\hat{y}(0) \hat{\phi}(0) \delta_{k}+O\left(\|\xi\|^{\kappa+1}\right), \quad \xi \rightarrow 0, \quad k \in \mathbb{Z}^{d} .
$$

Now by Proposition 4.3, the biorthogonal multiwavelet transform has $\kappa+1$ balancing order with respect to a vector conversion operator $E$ satisfying $E\left(\Pi_{\kappa}\right)=\mathcal{P}_{\kappa, Y}$ if and only if (6.3) holds. Hence, in the function setting, for biorthogonal multiwavelets, our notion of balancing order recovers that in [3, 4, 18, 19].

Moreover, by item (ii) of Proposition 4.2 and item (5) of Theorem 4.1 (i.e., $S_{a, M} \mathcal{P}_{\kappa, Y}=\mathcal{P}_{\kappa, Y}$ ), we must have $\mathcal{P}_{\kappa, y}=\mathcal{P}_{\kappa, Y}$. That is, by Lemma 2.6, there is a $2 \pi$-periodic trigonometric polynomial $\hat{c}$ with $\hat{c}(0) \neq 0$ such that $\hat{c}(\xi) \hat{y}(\xi)=$ $\hat{Y}(\xi)+O\left(\|\xi\|^{\kappa+1}\right)$ as $\xi \rightarrow 0$, which by Theorem 4.1 is also a necessary and sufficient condition for the biorthogonal multiwavelet to have $\kappa+1$ balancing order, since we assumed in advance that the mask $\hat{a}$ has $\kappa+1$ sum rules with $y \in\left(\ell_{0}\left(\mathbb{Z}^{d}\right)\right)^{1 \times r}$ in (2.2). Now it follows from (6.4) that

$$
\begin{aligned}
\hat{Y}(\xi) \hat{\phi}(\xi+2 \pi k) & =\hat{c}(\xi) \hat{y}(\xi) \hat{\phi}(\xi+2 \pi k)+O\left(\|\xi\|^{\kappa+1}\right) \\
& =\hat{y}(0) \hat{\phi}(0) \hat{c}(\xi) \delta_{k}+O\left(\|\xi\|^{\kappa+1}\right), \quad \xi \rightarrow 0, k \in \mathbb{Z}^{d} .
\end{aligned}
$$


In the time domain, by $\alpha_{j}=N^{-1} \omega_{j}$ for $j=0, \ldots, r-1$, the above relation is equivalent to

$$
\begin{gathered}
\sum_{j=1}^{r} \sum_{k \in \mathbb{Z}^{d}} h\left(k+\alpha_{j-1}\right) \phi_{j}(\cdot-k)=\sum_{j=1}^{r} \sum_{k \in \mathbb{Z}^{d}} h\left(k+N^{-1} \omega_{j-1}\right) \phi_{j}(\cdot-k) \\
=(h * Y) * \phi=(h * c * y) * \phi=\hat{y}(0) \hat{\phi}(0) h * c, \quad h \in \Pi_{\kappa} .
\end{gathered}
$$

The advantages of our approach are obvious. Even in the special case of biorthogonal multiwavelets, we do not assume in advance that $\hat{a}$ has $\kappa+1$ sum rules. Under the most natural condition (1.9), which is a necessary and sufficient condition for the perfect reconstruction in (1.20) of a multiframelet transform, our criterion and results on balancing orders of biorthogonal multiwavelets (or more generally $L=|\operatorname{det} M|-1)$ and dual multiframelets with an arbitrary number $L$ do not assume any other conditions. On the other hand, all other papers on balanced or prefiltered multiwavelets in the literature (see 1, 3, 4, 14, 15, 18, 19, 20) assumed that 1) there exist compactly supported $M$-refinable function vectors $\phi, \tilde{\phi}$ in $L_{2}\left(\mathbb{R}^{d}\right)$ satisfying (1.11) and the biorthogonality condition (1.15); 2) all the wavelet function vectors must have at least one vanishing moment in (1.14); 3) the mask $\hat{a}$ must have $\kappa+1$ sum rules. As we have seen above, these extra conditions in the function setting put some constraints on the masks $\hat{a}$ and $\hat{\tilde{a}}$. For example, as a consequence of these extra conditions, 1 must be a simple eigenvalue of $\hat{a}(0)$ and $\hat{\tilde{a}}(0)$ and all the other eigenvalues of $\hat{a}(0)$ and $\hat{\tilde{a}}(0)$ must be less than one in modulus. In the following, we shall present another example to show that our results apply equally to other cases which are excluded by the results on balancing orders in the literature even for the special case of $L=|\operatorname{det} M|-1$.

In some applications, it may be of interest to consider a more general vector conversion operator $E:\left(\ell\left(\mathbb{Z}^{d}\right)\right)^{1 \times s} \mapsto\left(\ell\left(\mathbb{Z}^{d}\right)\right)^{1 \times r}$ such that $E$ is a one-to-one and onto linear operator. Similarly, for $y \in\left(\ell_{0}\left(\mathbb{Z}^{d}\right)\right)^{s \times r}$, we define $\mathcal{P}_{\kappa, y}:=\{h * y:$ $\left.h \in\left(\Pi_{\kappa}\right)^{1 \times s}\right\} \subseteq\left(\Pi_{\kappa}\right)^{1 \times r}$. We point out that the analysis in this paper is applicable in this more general setting and most claims in this paper hold similarly, for example, for a vector conversion operator $E$ : $\left(\ell\left(\mathbb{Z}^{d}\right)\right)^{1 \times s} \mapsto\left(\ell\left(\mathbb{Z}^{d}\right)\right)^{1 \times r}$ such that $E\left(\left(\Pi_{\kappa}\right)^{1 \times s}\right)=\mathcal{P}_{\kappa, y}$ for some $y \in\left(\ell_{0}\left(\mathbb{Z}^{d}\right)\right)^{s \times r}$ such that $\hat{y}(0)$ has the full rank $s$. Then Theorem 4.1 still holds. More precisely, $T_{\tilde{a}, M} E\left(\left(\Pi_{\kappa}\right)^{1 \times s}\right) \subseteq E\left(\left(\Pi_{\kappa}\right)^{1 \times s}\right)$ and $T_{\tilde{b}^{\ell}, M} E\left(\left(\Pi_{\kappa}\right)^{1 \times s}\right)=0$ for all $\ell=1, \ldots, L$ if and only if (4.1) and (4.2) hold with $\hat{c}$ being an $s \times s$ matrix of $2 \pi$-periodic trigonometric polynomials. We shall address the balancing and prefiltering property of multiframelets in the more general setting as well as their symmetry and orthogonality properties in detail elsewhere. Here let us present the special case that $E:\left(\ell\left(\mathbb{Z}^{d}\right)\right)^{1 \times r} \mapsto\left(\ell\left(\mathbb{Z}^{d}\right)\right)^{1 \times r}$ is the identity mapping.

Corollary 6.1. Let $M$ be a $d \times d$ dilation matrix. Let $\hat{a}, \widehat{b^{1}}, \ldots, \widehat{b^{L}}$ and $\hat{\tilde{a}}, \widehat{\tilde{b}^{1}}, \ldots, \widehat{\tilde{b}^{L}}$ be $r \times r$ matrices of $2 \pi$-periodic trigonometric polynomials in $d$ variables such that (1.9) holds. Then the dual multiframelet transform satisfies $T_{\tilde{a}, M}\left(\Pi_{\kappa}\right)^{1 \times r} \subseteq$ $\left(\Pi_{\kappa}\right)^{1 \times r}$ and $T_{\tilde{b}^{\ell}, M}\left(\Pi_{\kappa}\right)^{1 \times r}=0$ for all $\ell=1, \ldots, L$ (that is, the associated multiframelet transform has $\kappa+1$ balancing order with respect to all vector conversion operators) if and only if $T_{\tilde{b}^{\ell}, M}\left(\Pi_{\kappa}\right)^{1 \times r}=0$ for all $\ell=1, \ldots, L$; that is,

$$
\widehat{\tilde{b}^{\ell}}(\xi)=O\left(\|\xi\|^{\kappa+1}\right), \quad \xi \rightarrow 0,
$$


for $\ell=1, \ldots, L$. If in addition $L=|\operatorname{det} M|-1$, then it is also equivalent to

$$
S_{a, M}\left(\Pi_{\kappa}\right)^{1 \times r} \subseteq\left(\Pi_{\kappa}\right)^{1 \times r} ;
$$

that is,

for all $\gamma \in \Gamma_{M^{T}} \backslash\{0\}$.

$$
\hat{a}(\xi+2 \pi \gamma)=O\left(\|\xi\|^{\kappa+1}\right), \quad \xi \rightarrow 0
$$

\section{REFERENCES}

[1] K. Attakitmongcol, D. P. Hardin, and D. M. Wilkes, Multiwavelet prefilters II: Optimal orthogonal prefilters, IEEE Trans. Image Proc. 10 (2001), 1476-1487.

[2] C. K. Chui, W. He and J. Stöckler, Compactly supported tight and sibling frames with maximum vanishing moments, Appl. Comput. Harmon. Anal. 13 (2002), 224-262. MR 1942743 (2004a:94011)

[3] C. K. Chui and Q. T. Jiang, Balanced multi-wavelets in $\mathbb{R}^{d}$, Math. Comp. 74 (2000), 13231344.

[4] C. K. Chui and Q. T. Jiang, Multivariate balanced vector-valued refinable functions. in Modern developments in multivariate approximation, 71-102, Internat. Ser. Numer. Math., 145, Birkhäuser, Basel, 2003. MR2070116

[5] I. Daubechies and B. Han, Pairs of dual wavelet frames from any two refinable functions, Constr. Approx., 20 (2004), 325-352. MR2057532 (2005d:42038)

[6] I. Daubechies, B. Han, A. Ron, and Z. Shen, Framelets: MRA-based constructions of wavelet frames, Appl. Comput. Harmon. Anal. 14 (2003), 1-46. MR1971300 (2004a:42046)

[7] B. Han, On dual wavelet tight frames, Appl. Comput. Harmon. Anal., 4 (1997), 380-413. MR.1474096 (98h:42031)

[8] B. Han, Approximation properties and construction of Hermite interpolants and biorthogonal multiwavelets, J. Approx. Theory 110 (2001), 18-53. MR.1826084 (2002e:41008)

[9] B. Han, Vector cascade algorithms and refinable function vectors in Sobolev spaces, J. Approx. Theory 124 (2003), 44-88. MR2010780 (2004h:42034)

[10] B. Han, Compactly supported tight wavelet frames and orthonormal wavelets of exponential decay with a general dilation matrix, J. Comput. Appl. Math., 155 (2003), 43-67. MR.1992289 (2004d:42064)

[11] B. Han, Dual multiwavelet frames with high balancing order and compact fast frame transform, Appl. Comput. Harmon. Anal. 26 (2009), 14-42. MR2467933

[12] B. Han, Symmetric orthonormal complex wavelets with masks of arbitrarily high linear-phase moments and sum rules, Adv. Comput. Math., d.o.i 10.1007/s10444-008-9102-7, to appear.

[13] B. Han and Q. Mo, Multiwavelet frames from refinable function vectors, Adv. Comput. Math. 18 (2003), 211-245. MR.1968120 (2004b:42079)

[14] D. P. Hardin and D. W. Roach, Multiwavelet prefilters I: Orthogonal prefilters preserving approximation order $p \leqslant 2$, IEEE Tran. Ciruits and System-II, 45 (1998), 1119-1125.

[15] J. Lebrub and M. Vetterli, Balanced multiwavelets: Theory and design, IEEE Trans. Signal Proc. 46 (1998), 1119-1125. MR1665663

[16] A. Ron and Z. Shen, Affine systems in $L_{2}\left(\mathbb{R}^{d}\right)$ II. Dual systems. J. Fourier Anal. Appl. 3 (1997), 617-637. MR1491938 (99g:42044)

[17] A. Ron and Z. Shen, Affine systems in $L_{2}\left(\mathbb{R}^{d}\right)$ : The analysis of the analysis operator. J. Funct. Anal. 148 (1997), 408-447. MR1469348 (99g:42043)

[18] I. W. Selesnick, Multiwavelet bases with extra approximation properties, IEEE Trans. Signal Proc. 46 (1998), 2898-2909. MR1719939 (2000g:94009)

[19] I. W. Selesnick, Balanced multiwavelet bases based on symmetric FIR filters, IEEE Trans. Signal Proc. 48 (2000), 184-191.

[20] X. G. Xia, D. P. Hardin, J. S. Geronimo, and B. Suter, Design of prefilters for discrete multiwavelet transforms, IEEE Trans. Signal Proc. 44 (1996), 25-35.

Department of Mathematical and Statistical Sciences, University of Alberta, Edmonton, Alberta, Canada T6G 2G1

E-mail address: bhan@math.ualberta.ca

$U R L:$ http://www.ualberta.ca/ bhan 